\title{
The WSRT wide-field H I survey
}

\section{Local Group features ${ }^{\star}$}

\author{
R. Braun ${ }^{1}$ and D. A. Thilker ${ }^{2}$ \\ 1 ASTRON, PO Box 2, 7990 AA Dwingeloo, The Netherlands \\ 2 Department of Physics and Astronomy, Johns Hopkins University, 3400 N. Charles St., Baltimore MD 21218-2695, USA
}

Received 30 September 2003 / Accepted 10 October 2003

\begin{abstract}
We have used the Westerbork array to carry out an unbiased wide-field survey of $\mathrm{HI}$ emission features, achieving an rms sensitivity of about $18 \mathrm{mJy} /$ Beam at a velocity resolution of $17 \mathrm{~km} \mathrm{~s}^{-1}$ over $1800 \mathrm{deg}^{2}$ and between $-1000<V_{\mathrm{Hel}}<$ $+6500 \mathrm{~km} \mathrm{~s}^{-1}$. The primary data consists of auto-correlation spectra with an effective angular resolution of 49' FWHM. The survey region is centered approximately on the position of Messier 31 and is Nyquist-sampled over $60 \times 30^{\circ}$ in RA $\times$ Dec. In this paper we present our H I detections at negative velocities which could be distinguished from the Galactic foreground. Fully $29 \%$ of the entire survey area has high velocity $\mathrm{HI}$ emission at a column density exceeding our $3 \sigma$ limit of about $1.5 \times 10^{17} \mathrm{~cm}^{-2}$ over $30 \mathrm{~km} \mathrm{~s}^{-1}$. We tabulate the properties of 95 discrete HVC components in the field, corresponding to more than an order of magnitude increase in number over that known previously. The distribution of discrete object number with flux density has an inflection point near $12 \mathrm{Jy}-\mathrm{km} \mathrm{s}^{-1}$ which may correspond to a characteristic mass and distance for this population. A faint population of discrete HVCs is detected in the immediate vicinity of M 31 which spans a large fraction of the M 31 rotation velocity. This class of features is confined to about $12^{\circ}(160 \mathrm{kpc})$ projected radius of M 31 and appears to be physically associated. Independent confirmation of the features within 3.5 (47 kpc) of M 31 has been obtained in our Green Bank Telescope survey (Thilker et al. 2004). We detect a diffuse northern extension of the Magellanic Stream (MS) from at least $\delta=+20 \rightarrow 40^{\circ}$, which subsequently loops back toward the south. Recent numerical simulations had predicted just such an MS extension corresponding to the apo-galacticon portion of the LMC/SMC orbit at a distance of $125 \mathrm{kpc}$. A faint bridge of $\mathrm{HI}$ emission appears to join the systemic velocities of M 31 with that of M 33 and continues beyond M 31 to the north-west. This may be the first detection of H I associated with the warm-hot intergalactic medium (WHIM). The distribution of peculiar velocity H I associated with M 31 can be described by a projected exponential of $25 \mathrm{kpc}$ scale-length and $5 \times 10^{17} \mathrm{~cm}^{-2}$ peak column density. We present the distribution function of $N_{\mathrm{HI}}$ in the extended $\mathrm{M} 31$ environment, which agrees well with the low red-shift QSO absorption line data over the range $\log \left(N_{\mathrm{HI}}\right)=17.2$ to $\log \left(N_{\mathrm{HI}}\right)=21.9$. Our data extend this comparison about two orders of magnitude lower than previously possible and provide the first image of the Lyman limit absorption system associated with an $\mathrm{L}_{*}$ Galaxy.
\end{abstract}

Key words. galaxies: Local Group - galaxies: evolution - galaxies: formation - galaxies: Magellanic Clouds galaxies: intergalactic medium - quasars: absorption lines

\section{Introduction}

The high velocity cloud (HVC) phenomenon has been under study for some 40 years, since the first detections of $\lambda 21 \mathrm{~cm}$ emission from atomic hydrogen at velocities far removed from those allowed by rotation in the Galaxy disk (Muller et al. 1963). Several explanations have been put forth for their interpretation, including a galactic fountain, infall of circumgalactic gas, tidal debris from mergers and sub-galactic-mass companions. The suggestion has been made that at least one

Send offprint requests to: $\mathrm{R}$. Braun, e-mail: rbraun@astron. $\mathrm{nl}$

* Table 2 is only available in electronic form at the CDS via anonymous ftp to cdsarc.u-strasbg.fr $(130.79 .128 .5)$ or via http://cdsweb.u-strasbg.fr/cgi-bin/qcat?]/A+A/417/421 component of the Galactic HVCs, the so-called CHVCs (Braun \& Burton 1999; Blitz et al. 1999) might be the gaseous counterpart of low-mass dark-matter satellites. A critical prediction of this scenario (De Heij et al. 2002c) is that a large population of faint CHVCs should be detected in the vicinity of M 31 (at declination $+40^{\circ}$ ) if enough sensitivity were available. While existing observational data were consistent with this scenario, they were severely limited by the modest point source sensitivity available at northern declinations (within the Leiden/Dwingeloo Survey, Hartmann \& Burton 1997) which is almost an order of magnitude poorer than that of HIPASS (Barnes et al. 2001) in the south.

We have undertaken a moderately sensitive large-area $\mathrm{HI}$ survey both to test for the predicted population of faint CHVCs 
near M 31 as well as to carry out an unbiased search for H I emission associated with background galaxies. We have achieved an rms sensitivity of about $18 \mathrm{mJy} /$ Beam at a velocity resolution of $17 \mathrm{~km} \mathrm{~s}^{-1}$ over $1800 \mathrm{deg}^{2}$ and between $-1000<V_{\mathrm{Hel}}<+6500 \mathrm{~km} \mathrm{~s}^{-1}$. The corresponding rms column density sensitivity for emission filling the $3000 \times 2800$ arcsec effective beam area is about $4 \times 10^{16} \mathrm{~cm}^{-2}$ over $17 \mathrm{~km} \mathrm{~s}^{-1}$. For comparison, the HIPASS survey has achieved an rms of about $14 \mathrm{mJy} /$ Beam at a velocity resolution of $18 \mathrm{~km} \mathrm{~s}^{-1}$, yielding a slightly superior flux sensitivity. On the other hand, the column density sensitivity for emission filling our larger beam exceeds that of HIPASS by almost an order of magnitude. Since the linear FWHM diameter of our survey beam varies from about $10 \mathrm{kpc}$ at a distance of $0.7 \mathrm{Mpc}$ to more than $1 \mathrm{Mpc}$ at $75 \mathrm{Mpc}$, it is only at Local Group distances that the condition of beam filling is likely to be achieved. Compared to the Leiden/Dwingeloo Survey, we achieve an order of magnitude improvement in both flux density and brightness sensitivity. We detect more than 100 distinct features at high significance in each of the two velocity regimes (negative and positive LGSR velocities).

In a previous paper (Braun et al. 2003, hereafter referred to as BTW03) we have described the survey observations and general data reduction procedures, together with the results for our H I detections of external galaxies. In this paper, we briefly relate some additional data reduction procedures in Sect. 2 and present the results in Sect. 3. In addition to a large sample of discrete HVC components we detect three diffuse complexes of peculiar velocity gas. We discuss these results in Sect. 4 and close with a summary in Sect. 5 .

\section{Observations and data reduction}

A complete description of the observations and general methods of data reduction has already been given in BTW03, so we will only summarize the essential points here and indicate any departures from the methods employed for the positive velocity portion of the survey.

\subsection{Observations}

The survey area has an extent of $60 \times 30$ true degrees oriented in $\alpha_{2000} \times \delta_{2000}$ and centered on $\left(\alpha_{2000}, \delta_{2000}\right)=\left(10^{\circ}, 35^{\circ}\right)$, about $5^{\circ}$ south of the M 31 nuclear position. Auto-correlation (and cross-correlation) spectra were acquired with one minute data averaging using the Westerbork Synthesis Radio Telecope (WSRT) in a series of 120 RA drift-scans separated by $15^{\prime}$ in declination, providing Nyquist sampling of the $35^{\prime}$ FWHM primary beam. Both linear polarizations were recorded over two $20 \mathrm{MHz}$ bands centered at geocentric frequencies of 1416 and $1398 \mathrm{MHz}$ with 512 uniformly weighted spectral channels per band. A hanning smoothing was applied after the fact to minimize the spectral side-lobes of interference, yielding a spectral resolution of $78.125 \mathrm{kHz}$, corresponding to about $16.6 \mathrm{~km} \mathrm{~s}^{-1}$ over a heliocentric velocity range of about $-1000<V_{\mathrm{HEL}}<$ $+6500 \mathrm{~km} \mathrm{~s}^{-1}$.

\subsection{Data reduction}

Data editing, calibration and gridding were employed to generate a data-cube of the survey volume as described in BTW03. Applying the same processing steps to a digital representation of the holographically determined telescope primary beam allowed estimation of the effective beam pattern of the survey. The central lobe of this effective beam can be approximated by a Gaussian of $3020 \times 2810 \operatorname{arcsec}$ at $\mathrm{PA}=90^{\circ}$. The maximum near-in side-lobe levels are only about $0.25 \%$ of the peak response and are concentrated in an " $X$ " pattern $(\mathrm{PA}= \pm 45$, $\pm 135^{\circ}$ ) that is due to the four feed-support legs. Beyond a radius of about $3^{\circ}$ sidelobe levels fall below $0.05 \%$ even along the " $X$ ". Since the telescopes of the array are equatorially mounted, the side-lobe pattern is fixed relative to the sky. This is an important simplifying factor for the succesful modeling of the beam. Although moderately faint, the side-lobe response pattern can be easily distinguished adjacent to the bright emission regions of M 31 and M 33. An attempt was made to deconvolve the data-cube with the effective beam model using the APCLN task of the Classic AIPS package. While moderately successful, this procedure did leave some residual artifacts at a level below about $0.1 \%$ within a $3^{\circ}$ radius of the brightest emission regions. An illustration of the "dirty" and "clean" channel maps is given in Fig. 1. The sense of the clean residual artifact noted above is a slight over-subtraction of the near-in sidelobes. This will result in a small, systematic underestimate of the column density of any diffuse emission features near M 31 and M 33. The likely reason for this over-subtraction effect lies in the adopted method of local spatial and velocity baselining that was employed in calibrating the data. Extremely faint features will not have been excluded from the sub-set of data that was used for local spatial and velocity baselining, leading to their suppression. This is likely to remain a challenging aspect of deep wide-field imaging in total power. The faint positive extensions from the bright M 31 disk seen in Fig. 1 are not artifacts, but have been confirmed as real emission features in our recent imaging with the Green Bank Telescope (Thilker et al. 2004). The rms noise level in the final data-cube was $17.4 \mathrm{mJy} /$ Beam over $16.6 \mathrm{~km} \mathrm{~s}^{-1}$ at negative radial velocities $\left(>-1000 \mathrm{~km} \mathrm{~s}^{-1}\right)$ which were unconfused by emission from the Galaxy. The corresponding rms in H I column density was $3.8 \times 10^{16} \mathrm{~cm}^{-2}$ for emission filling the $3020 \times 2810 \operatorname{arcsec}$ beam.

After deconvolution, a grid of smoothed data-cubes was generated at velocity resolutions of 18,36 and $72 \mathrm{~km} \mathrm{~s}^{-1}$ $F W H M$ and spatial resolutions of 48,63 and $97^{\prime} F W H M$, to allow optimized detection of diffuse features. Smoothed datacubes were used as filtering masks to isolate regions of $\mathrm{HI}$ emission from the noise background in cubes at a higher resolution. Typical masking thresholds of $2 \sigma$ were employed in the smoothed cubes in order to optimize signal-to-noise in the images of integrated emission by minimizing the velocity interval of the integration. The resulting data-cubes have been resampled in velocity onto various systems: including heliocentric (HEL), Local Standard of Rest (LSR), Galactic Standard 

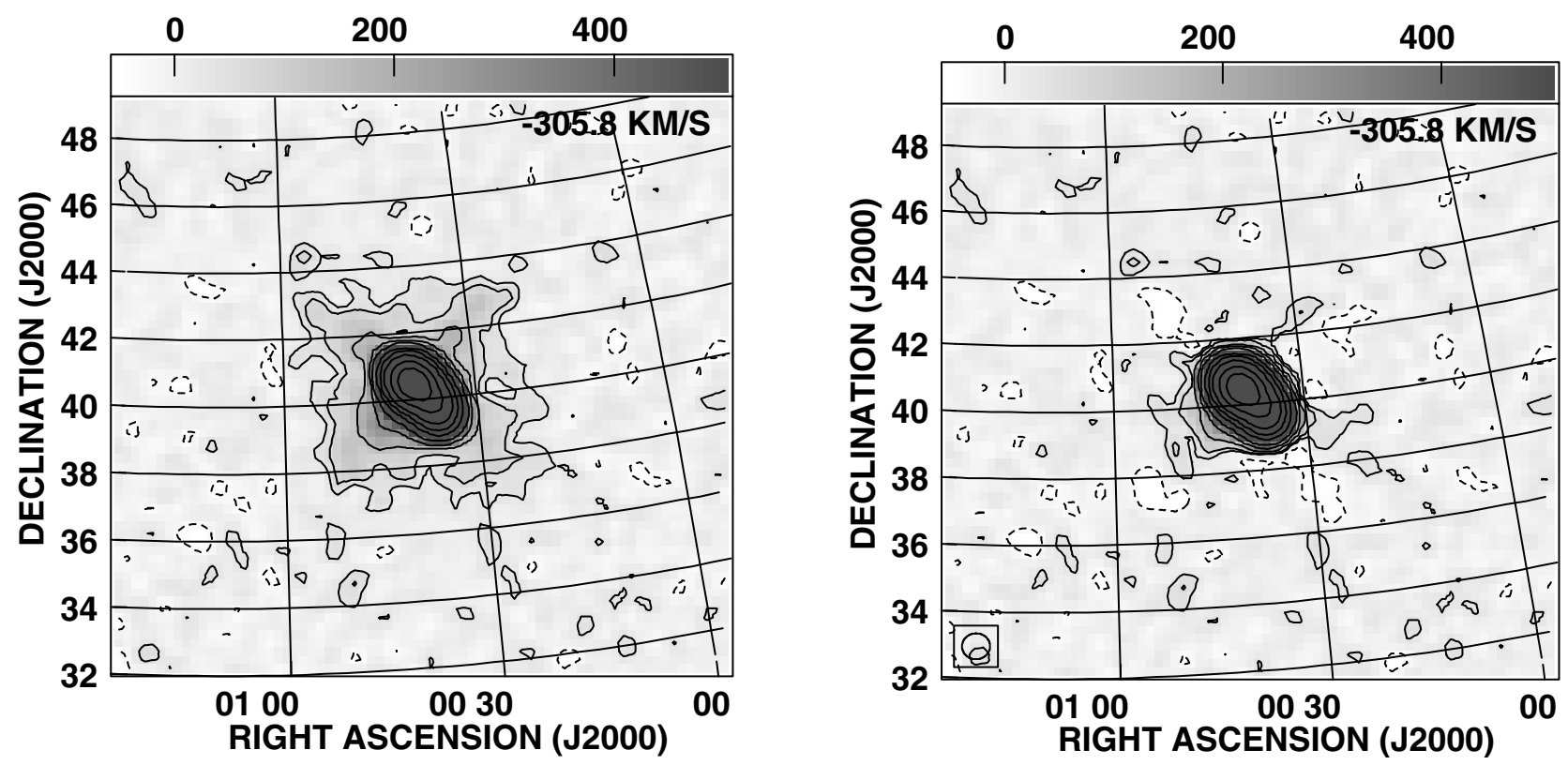

Fig. 1. Illustration of a "dirty" and "clean" channel map. H I emission from a $17 \times 17^{\circ}$ sub-field centered on M 31 at $V_{\mathrm{LGSR}}=-306 \mathrm{~km} \mathrm{~s}{ }^{-1}$ both before (left) and after (right) deconvolution with the effective auto-correlation beam. The linear grey-scale extends from -50 to $500 \mathrm{mJy} / \mathrm{Beam}$, while contours are drawn at $-1,1,2,5,10,20,50,100,200,500$ and 1000 times $30 \mathrm{mJy} /$ Beam. The peak brightness in this channel is about $60 \mathrm{Jy} /$ Beam. Note the " $X$ "-shaped pattern of side-lobes that is visible out to a radius of about $3^{\circ}$ in the "dirty" image. The dynamic range in the "clean" image is substantially improved, although there is a systematic over-subtraction of the near-in sidelobes, as discussed in the text.

of Rest (GSR) and Local Group Standard of Rest (LGSR) defined by:

$V_{\mathrm{LSR}}=V_{\mathrm{HEL}}+9 \cos (l) \cos (b)+12 \sin (l) \cos (b)+7 \sin (b)$

$V_{\mathrm{GSR}}=V_{\mathrm{LSR}}+0 \cos (l) \cos (b)+220 \sin (l) \cos (b)+0 \sin (b)$

$V_{\mathrm{LGSR}}=V_{\mathrm{GSR}}-62 \cos (l) \cos (b)+40 \sin (l) \cos (b)-35 \sin (b)$. (3)

\section{Results}

The integrated H I emission of all negative velocity features in our survey field which could be unambiguously distinguished from the foreground emission of our Galaxy are depicted in Fig. 2. Our criterion for distinguishing such negative velocity features from the foreground Galactic emission was the detection of a local peak in the emission spectrum in contrast to merely an extended wing of emission having a spectral peak at a velocity allowed by Galactic rotation. While only a handful of objects were known previously in this region; namely M 31, M 33, Davies' Cloud (Davies 1975), Wright's Cloud (Wright 1979), and 9 additional high velocity clouds (De Heij et al. 2002a) all tabulated in Table 1, we detect high velocity $\mathrm{HI}$ emission at a column density in excess of our $3 \sigma$ limit of about $1.5 \times 10^{17} \mathrm{~cm}^{-2}$ from fully $29 \%$ of the area of our $1800 \mathrm{deg}^{2}$ survey field. The detected emission is due to a mix of discrete and diffuse components.

\subsection{A catalog of high velocity clouds}

We have tabulated the properties of all local maxima with a peak column density (at the full survey resolution) in excess of $5 \sigma$ in Table 2 . Only the four previously known objects at the top of Table 1 were excluded from this tabulation. The 95 additional high velocity features we detect in the survey field correspond to an order of magnitude increase in number over that seen before. We have classified each component as a CHVC (compact high velocity cloud) or an HVC (high velocity cloud) based on the degree of isolation in an image of integrated H I emission made over the full velocity extent of the particular object in question. Objects which were not connected to other features at a level exceeding $1.5 \times 10^{17} \mathrm{~cm}^{-2}$ (about $3 \sigma$ ) in this image were deemed isolated and were given the CHVC designation, while those objects that were connected to other features at this level are given the HVC designation in the Table. The majority of the tabulated features (79 of 95) are classified as isolated by this criterion. Previous tabulations of allsky CHVC and HVC populations (Putman et al. 2002; De Heij et al. 2002a) have used an isolation criterion based on a column density of about $1.5 \times 10^{18} \mathrm{~cm}^{-2}$ that was matched to the lower column density sensitivities of the HIPASS and LDS surveys (also about $3 \sigma$ ). The position and size of each component has been obtained from a 2-D spatial Gaussian fit to an image of integrated emission. No correction was made for the finite beam size of the survey. The peak column density, centroid velocity and $F W H M$ line-width were determined from a Gaussian fit to the single spectrum with the maximum column density. An estimate of the total H I flux density for each component was made from the product of peak column density with the spatial extent normalized by the beam area.

The columns of Table 2 denote the following:

Column 1: running identifying number in the catalog.

Column 2: designation, consisting of a prefix, followed by the Galactic longitude, Galactic latitude, and Local 




Fig. 2. Integrated negative velocity $\mathrm{H}$ I within the survey region at $48^{\prime}, 18 \mathrm{~km} \mathrm{~s}^{-1}$ resolution. The masked distribution of negative velocity $\mathrm{HI}$ that is distinct from Galactic IVCs has been integrated and converted to an H I column density under the assumption of negligible opacity in the $\lambda 21 \mathrm{~cm}$ line. The grey-scale varies between $\log \left(N_{\mathrm{HI}}\right)=17-18$, for $N_{\mathrm{HI}}$ in units of $\mathrm{cm}^{-2}$. Contours are drawn at $\log \left(N_{\mathrm{HI}}\right)=17,17.5,18, \ldots 20.5$.

Table 1. Previously detected objects in the survey field.

\begin{tabular}{lccrrrr}
\hline \hline Name & $\begin{array}{c}\mathrm{RA}_{2000} \\
(\mathrm{~h} \mathrm{~m})\end{array}$ & $\begin{array}{c}\mathrm{Dec}_{2000} \\
\left({ }^{\prime}\right)\end{array}$ & $\begin{array}{r}V_{\text {HEL }} \\
\left(\mathrm{km} \mathrm{s}^{-1}\right)\end{array}$ & $\begin{array}{r}V_{\mathrm{GSR}} \\
\left(\mathrm{km} \mathrm{s}^{-1}\right)\end{array}$ & $\begin{array}{r}V_{\mathrm{LGSR}} \\
\left(\mathrm{km} \mathrm{s}^{-1}\right)\end{array}$ & $\begin{array}{r}W_{20} \\
\left(\mathrm{~km} \mathrm{~s}^{-1}\right)\end{array}$ \\
$(1)$ & $(2)$ & $(3)$ & $(4)$ & $(5)$ & $(6)$ & $(7)$ \\
\hline Messier 31 & 0043 & +4116 & -300 & -122 & -34 & 536 \\
Messier 33 & 0134 & +3040 & -179 & -44 & +37 & 199 \\
Davies' Cloud & 0038 & +4228 & -446 & -265 & -176 & 38 \\
Wright's Cloud & 0115 & +2900 & -423 & -259 & -200 & 120 \\
HVC091.8-35.6-393 & 2305 & +2043 & -398 & -215 & -160 & 75 \\
CHVC107.7-29.7-429 & 2349 & +3119 & -440 & -247 & -180 & 61 \\
CHVC108.3-21.2-402 & 2340 & +3940 & -407 & -208 & -141 & 50 \\
CHVC118.5-32.6-386 & 0034 & +3005 & -388 & -223 & -149 & 40 \\
HVC118.7-31.1-135 & 0034 & +3137 & -137 & +30 & +104 & 29 \\
CHVC119.2-31.1-384 & 0036 & +3139 & -386 & -220 & -146 & 29 \\
CHVC122.9-31.8-325 & 0051 & +3104 & -326 & -168 & -93 & 52 \\
HVC127.5-41.6-341 & 0106 & +2109 & -339 & -210 & -135 & 66 \\
HVC147.0-33.9-125 & 0219 & +2450 & -120 & -26 & +55 & 107 \\
\hline
\end{tabular}

Standard of Rest velocity. The prefix is CHVC for the clouds satisfying our isolation criteria; while HVC is used to indicate clouds connected to more extended complexes. The longitude and latitude refer to the peak of a spatial Gaussian fit to the integrated HI. The velocity refers to the peak of a Gaussian fit to the single spectrum of peak column density.

Columns 3 and 4: J2000 right ascension and declination of the position listed in Col. 2.

Columns 5: heliocentric radial velocities. 


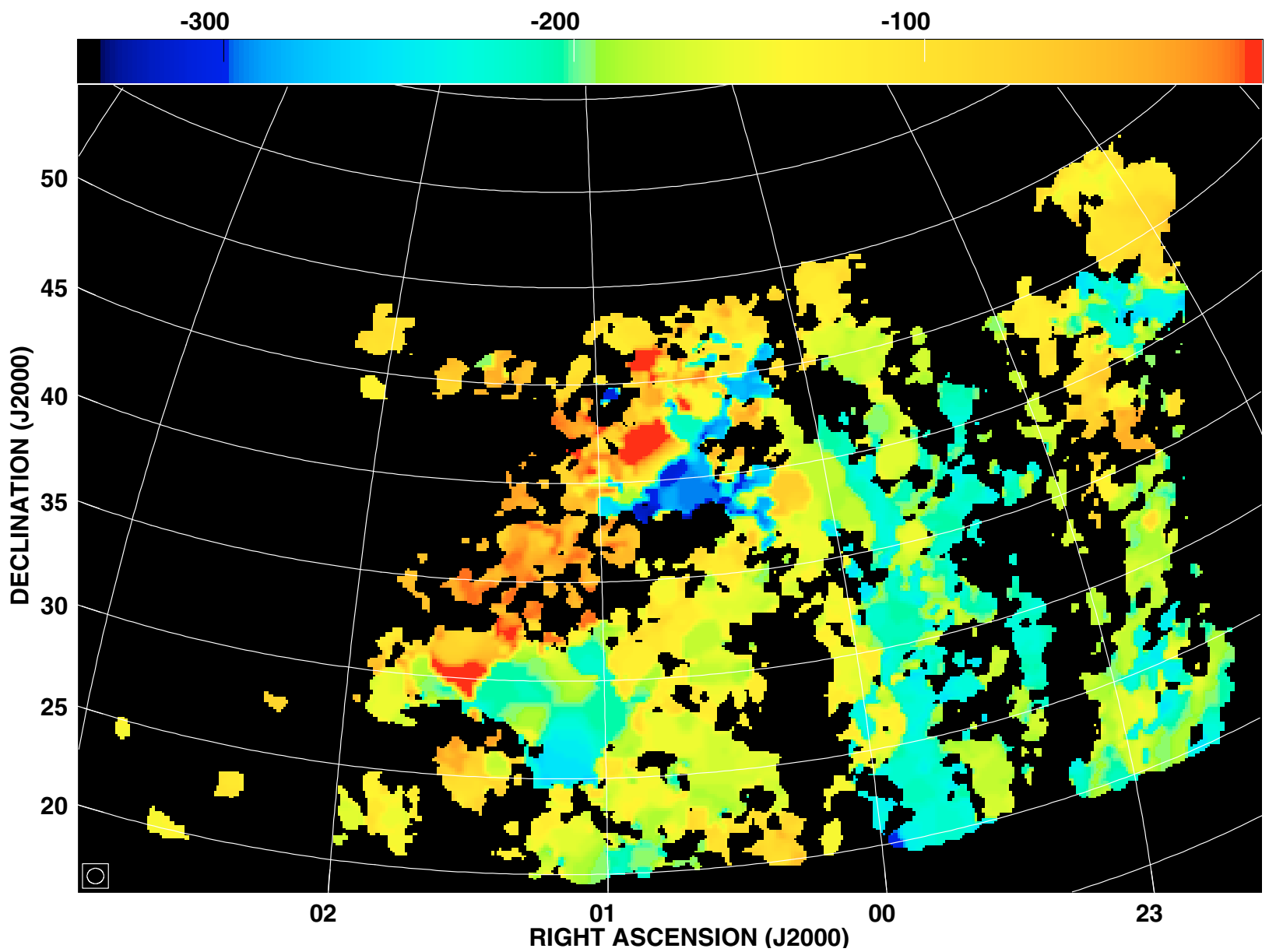

Fig. 3. Radial velocity in the LGSR frame of all detected H I features at negative velocity at $48^{\prime}, 18 \mathrm{~km} \mathrm{~s}^{-1}$ resolution. The color wedge varies linearly from -340 to $-4 \mathrm{~km} \mathrm{~s}^{-1}$ and corresponds to the velocity of the brightest emission feature along each line-of-sight. Note the continuous variation of velocity with position within the major diffuse features (the Magellanic Stream, Wright's Cloud and the M 31/M 33 bridge) yet the large velocity offsets between them.

Column 6: velocity FWHM of the single spectrum with peak column density.

Column 7: peak column density in units of $10^{18} \mathrm{~cm}^{-2}$.

Columns 8-10: angular FWHM of the major and minor axes, and major axis position angle. These are the results of a spatial Gaussian fit to an image of integrated H I, with position angle expressed in the (RA, Dec) frame.

Column 11: total flux of the detection, in units of Jy-km s${ }^{-1}$. Column 12: catalog ID's prefaced with DBB refer to the entries of Table 1 of De Heij et al. 2002a, those prefaced with BB to Table 1 of Braun \& Burton 1999 and those with WW to Table 1 of Wakker \& van Woerden 1991.

\subsection{Kinematically distinct complexes of high velocity gas}

The line-of-sight velocities, in the LGSR system, which accompany all detected features are depicted in Fig. 3. Rather than depicting the first moment of the distribution, $V_{\text {Mom }}$, we have chosen to depict the velocity of the brightest emission feature seen along each line-of-sight, $V_{\mathrm{Pk}}$. This was done since multiple velocity components are present in many directions. Such circumstances are more easily recognized by the sudden changes in line-of-sight velocity which are preserved in $V_{\mathrm{Pk}}$ while being smeared out in $V_{\mathrm{Mom}}$. There are several large-scale patterns in the velocity field which permit classification of the detected features into a small number of categories:

1. A widespread component of diffuse emission is visible over much of the western half of the survey field in the form of a filament extending north-north-west from $(\alpha, \delta)=$ $(23: 00,+20)$ to $(22: 30,+35)((l, b)=(90,-36)$ to $(93$, $-19))$ and a filamentary loop extending to the north from $(\alpha, \delta)=(23: 40,+20)$ to $(23: 40,+42)$ and arcing back down to $(01: 00,+20)((l, b)=(101,-40)$ to $(109,-19)$ and back to $(126,-43))$. LGSR velocities vary smoothly along these structures from about -250 to $-150 \mathrm{~km} \mathrm{~s}^{-1}$. To the north of the western-most filament are additional diffuse features at a number of discrete velocities ranging from -250 to $-50 \mathrm{~km} \mathrm{~s}^{-1}$. Against the diffuse filamentary background are a large number of condensations at various LGSR velocities. Some of these are presumably associated with the diffuse features, while others appear at distinct velocities and show no morphological connection to the diffuse filaments. As we will show below, these diffuse filamentary 
features are a previously undetected northern extension of the Magellanic Stream (MS).

2. The Local Group galaxies M $31\left(V_{\mathrm{sys}}=-34 \mathrm{~km} \mathrm{~s}^{-1}\right.$ in the LGSR frame) and M $33\left(V_{\text {sys }}=+37 \mathrm{~km} \mathrm{~s}^{-1}\right)$ are visible in the central portion of the survey field, together with a diffuse bridge of emission that extends between them and continues to the north-west of M 31 to $(\alpha, \delta)=(00: 20$, $+48)$. The radial velocity varies along this feature from the M 31 systemic velocity near that galaxy down to $V_{\text {LGSR }}=$ $0 \mathrm{~km} \mathrm{~s}^{-1}$ toward M 33. The bridge feature can not be traced effectively to positive LGSR velocities due to confusing foreground emission from the Galaxy. The full velocity interval corresponding to rotation of $\mathrm{M} 31\left(-330>V_{\mathrm{LGSR}}>\right.$ $\left.+270 \mathrm{~km} \mathrm{~s}^{-1}\right)$ and M $33\left(-85>V_{\text {LGSR }}>+150 \mathrm{~km} \mathrm{~s}^{-1}\right)$ is also truncated at $V_{\mathrm{LGSR}}=0 \mathrm{~km} \mathrm{~s}^{-1}$ for the same reason. Nonetheless, a population of faint, discrete features can be seen near M 31, spanning much of the range of M 31 rotation velocities that are unconfused. Only within a radius of about 12 degrees of M 31 are such a wide variety of radial velocities detected. Many of these discrete features have been verified in independent observations with the Green Bank Telescope (Thilker et al. 2004). Both the discrete and diffuse populations near M 31 appear distinct from the Magellanic Stream features described above. The M 31/M 33 bridge is offset from the nearest MS filament by about 10 degrees on the sky and about $100 \mathrm{~km} \mathrm{~s}^{-1}$ in radial velocity.

3. A third component, Wright's Cloud and extensions, is seen in the south-central portion of Fig. 3. Although partially overlapping both the Magellanic Stream and M 33 in projection, this feature has quite distinctive kinematics. As seen in the figure, radial velocities are offset by more than $100 \mathrm{~km} \mathrm{~s}^{-1}$ from the M 33 and to a lesser extent from the MS. Added to this are exceptional internal kinematics which we discuss below.

\section{Discussion}

\subsection{Discrete HVC components}

The statistical properties of the discrete high velocity components listed in Table 2 are illustrated in Fig. 4. The distributions of detected flux density and peak column density are both rising to lower values. These distributions can be compared to those found previously in the LDS survey (De Heij et al. 2002a) and at negative declinations in the HIPASS survey (Putman et al. 2002). A linear regression solution for the logarithmically binned flux density (above $20 \mathrm{Jy}-\mathrm{km} \mathrm{s}^{-1}$ ) has slope -1.15 and for the peak column density (above $10^{18} \mathrm{~cm}^{-2}$ ) has slope -1.07 . The corresponding distribution functions in linear units can be expressed as $f\left(S_{\mathrm{HI}}\right) \propto S_{\mathrm{HI}}^{-2.15}$ and $f\left(N_{\mathrm{HI}}\right) \propto N_{\mathrm{HI}}^{-2.07}$. The linearly binned distribution functions are also shown in the figure with the power-law solutions overlaid. Our flux density distribution agrees well, in terms of both the high-end slope and the apparent low-end turn-over, with that seen for the HVC population detected in the HIPASS data.

The apparent column density distributions, on the other hand, are much more discrepant. Our high-end slope of -1.07 is much flatter than the -1.9 seen in the HIPASS sample, with the apparent mode shifted to lower column density by about an order of magnitude. The very different beam size and limiting column density of the two surveys (48' versus $16^{\prime}$ and $2 \times 10^{17}$ versus $2 \times 10^{18} \mathrm{~cm}^{-2}$ ) is likely to play an important role in accounting for this difference. The apparent mode of the observed $F W H M$ size in the HIPASS survey is only about $38^{\prime}$, while the mode for the WSRT wide-field survey is $60^{\prime}$. Both of these values are consistent with a typical intrinsic angular FWHM size which is smaller than the beamwidth. A more quantitative comparison is complicated by the need to make assumptions about the intrinsic radial variation of column density within individual features. High resolution total power imaging of a sample of CHVCs (Burton et al. 2001) with the 3.5 Arecibo telescope beam has demonstrated approximately exponential column density profiles of these objects with radius. Such profiles have the appearance of being marginally resolved by essentially any size of telescope beam with which they are observed. If such column density profiles are the norm, then it is likely that both the HIPASS and current survey underestimate the intrinsic peak column density of discrete features.

The distribution of velocity line-width is strongly peaked at a $F W H M$ of $32 \mathrm{~km} \mathrm{~s}^{-1}$, with a lower cut-off of $20 \mathrm{~km} \mathrm{~s}^{-1}$ and a sparsely populated tail extending out to more than $100 \mathrm{~km} \mathrm{~s}^{-1}$. High spatial and velocity resolution imaging (Braun \& Burton 2000; Burton et al. 2001; De Heij et al. 2002c) has demonstrated that the global spectrum of such features is composed of a narrow component due to cool (about $100 \mathrm{~K}$ ) gas accounting for between 1 and $50 \%$ of the total flux, a broader component (consistent with $10^{4} \mathrm{~K}$ temperature) accounting for the remainder of the H I flux, together with the possibility of relative motions of internal sub-structures varying from 0 to $100 \mathrm{~km} \mathrm{~s}^{-1}$. The mode of the line-width distribution, near $32 \mathrm{~km} \mathrm{~s}^{-1}$, is consistent with only modest kinematic broadening of a predominantly warm (about $10^{4} \mathrm{~K}$ ) H I phase.

Both the observed size and line-width distributions agree very well with those seen in the LDS survey (De Heij et al. 2002a) which had comparable angular resolution, but much higher velocity resolution (of $1 \mathrm{~km} \mathrm{~s}^{-1}$ ). Extremely narrow global line profiles are thus rather rare for high velocity $\mathrm{H} \mathrm{I}$ features.

The completeness of our HVC tabulation is a complicated function of position and velocity. De Heij et al. (2002b) have shown that obscuration by Galactic HI, coupled with the intrinsic spatial and kinematic deployment, play a crucial role in determining which fraction of features can be detected. At velocities free of Galactic obscuration, our rms noise level is quite uniform. Since both the size and line-width distributions of detected features are strongly peaked, it is then straightforward to estimate the typical error in integrated flux density, $\sigma_{\text {Typ }}=0.66 \mathrm{Jy}-\mathrm{km} \mathrm{s}^{-1}$ (for a $60^{\prime}, 32 \mathrm{~km} \mathrm{~s}^{-1}$ apparent source extent). This is a factor of about 2.2 higher than the error for features unresolved both spatially and in velocity, $\sigma_{\mathrm{Un}}=$ $0.29 \mathrm{Jy}^{-\mathrm{km} \mathrm{s}^{-1}}$ (for a $48^{\prime}, 17 \mathrm{~km} \mathrm{~s}^{-1}$ resolution). Simulations involving the injection of artificial sources into total power surveys for external galaxies (e.g. Rosenberg \& Schneider 2002) suggest that an asymptotic value of completeness is achieved above a signal-to-noise ratio of about $8-10$ in total Hi flux. 

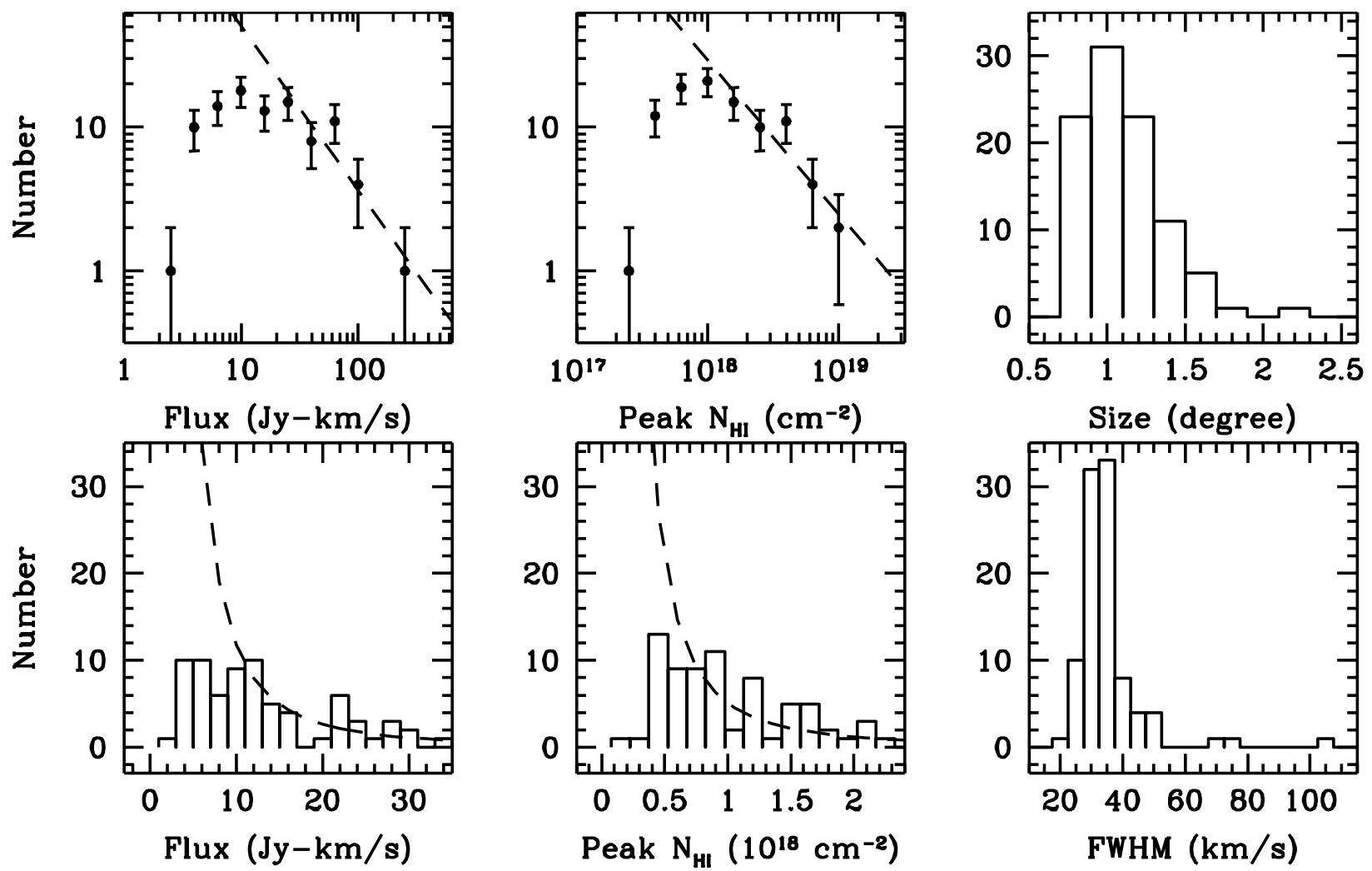

Fig. 4. Population properties of discrete HVC components. Histograms of integrated flux density (left panels), peak column density (center panels), average FWHM size (top right) and velocity FWHM (bottom right) are shown for the entries of Table 2. The linear regression solution for the flux density (above $20 \mathrm{Jy}-\mathrm{km} \mathrm{s}^{-1}$ ) has slope -1.15 and for the peak column density (above $10^{18} \mathrm{~cm}^{-2}$ ) has slope -1.07 . The corresponding distribution functions in linear units have power-law indices of -2.15 and -2.07 and are overlaid in the bottom panels.

By this criterion, we would expect a high degree of completeness (at unconfused velocities) for those features exceeding about $S_{\text {Comp }}=6 \mathrm{Jy}-\mathrm{km} \mathrm{s}^{-1}$. Interestingly, the number distribution of flux density shown in the bottom left panel of Fig. 4 is essentially flat between about 6 and $12 \mathrm{Jy}-\mathrm{km} \mathrm{s}^{-1}$ and only becomes steeper at higher flux densities. The inflection point in the distribution near $S_{\text {Inf }}=12 \mathrm{Jy}-\mathrm{km} \mathrm{s}^{-1}$ may be an indication that our survey sensitivity is sufficient to detect HVC features out to some characteristic distance beyond which their space number density diminishes dramatically.

As indicated above, a similar turn-over of the logarithmically binned flux density distribution is also seen in the HIPASS sample (Putman et al. 2002). The HIPASS HVC flux error for unresolved sources (at 16', $26 \mathrm{~km} \mathrm{~s}^{-1}$ resolution) is about $0.34 \mathrm{Jy}-\mathrm{km} \mathrm{s}^{-1}$, while for the typical observed source extent of $38^{\prime}$ and $32 \mathrm{~km} \mathrm{~s}^{-1}$ this increases to about $2.1 \mathrm{Jy}-\mathrm{km} \mathrm{s}^{-1}$. A high degree of completeness is then expected in the HIPASS HVC sample only above about $20 \mathrm{Jy}-\mathrm{km} \mathrm{s}^{-1}$. The similar turn-over of the HIPASS and WSRT wide-field distributions of flux density below $20 \mathrm{Jy}-\mathrm{km} \mathrm{s}^{-1}$ must therefore be considered fortuitous.

The velocity coverage within our survey region, $-1000<$ $V+6500 \mathrm{~km} \mathrm{~s}^{-1}$, appears to have been more than sufficient to encompass all likely instances of high velocity $\mathrm{HI}$ associated with the Local Group. All of our HVC detections are at negative LSR velocities, which is in keeping with the previously known HVC properties at these Galactic longitudes (cf. De Heij et al. 2002a). Our negative velocity coverage is more extensive than that of the LDS survey $\left(-450<V_{\mathrm{LSR}}+\right.$ $400 \mathrm{~km} \mathrm{~s}^{-1}$ ) and in fact we detect several features at more extreme LSR velocities than any which were previously seen. The previous record holder was the object CHVC110.6-08.0-466, discovered by Hulsbosch (1978). This has been surpassed, in the general survey field (excluding the immediate vicinity of M 31), by the object CHVC093.4-11.9-485.

In the immediate vicinity of $\mathrm{M} 31$, an entire population of very faint objects has been detected in our $7 \times 7^{\circ}$ Green Bank Telescope (GBT) survey of Thilker et al. (2004) extending out to $-520 \mathrm{~km} \mathrm{~s}^{-1}$. The most extreme velocity object in our tabulation which belongs to this class, but is still distinct from the diffuse M 31 disk emission features at our survey angular resolution, is CHVC115.4-23.1-503. With an integrated H I flux of only $2.8 \mathrm{Jy}-\mathrm{km} \mathrm{s}^{-1}$, this is the faintest object in our catalog. At the distance of $\mathrm{M} \mathrm{31}$, this would correspond to an $M_{\mathrm{HI}}=4 \times 10^{5} M_{\odot}$. Our $8 \sigma$ completeness limit for a spatially unresolved, $32 \mathrm{~km} \mathrm{~s}^{-1}$ source extent corresponds to about $4.5 \times 10^{5} M_{\odot}$ at the M 31 distance.

\subsection{The magellanic steam}

An important component of the high velocity H I gas associated with the Galaxy is that due to the interaction of the Magellanic Clouds with one another as well as with the Galaxy. This feature was first assocated with the Magellanic Clouds by Mathewson et al. (1974) and has most recently been discussed 


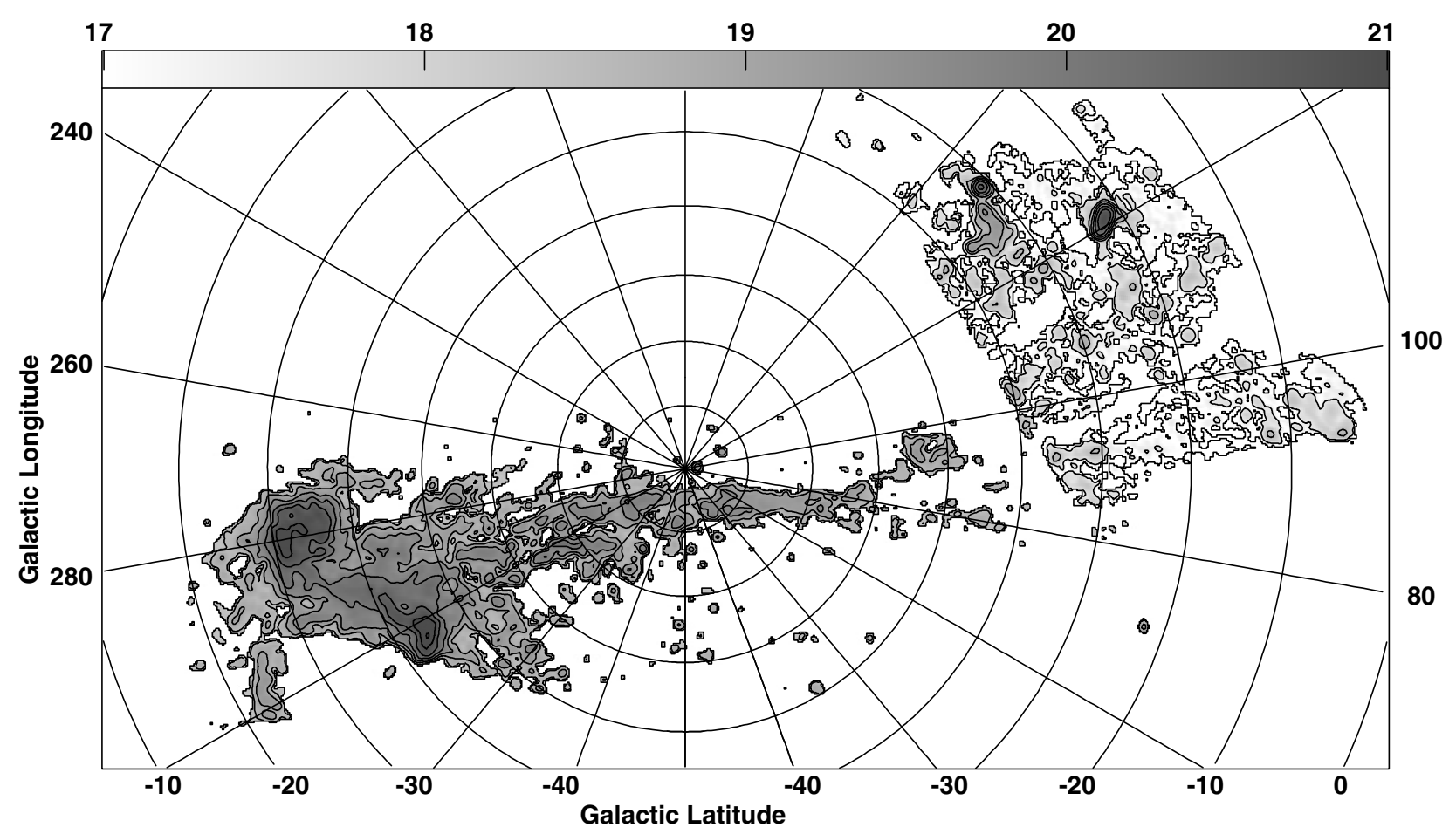

Fig. 5. Integrated H I emission image highlighting the Magellanic Clouds and Stream. The HIPASS data of Putman et al. (2003) is combined with the wide-field WSRT survey data (in the region $90<l<160,-40<b<-5$ ). The grey-scale varies between $\log \left(N_{\mathrm{HI}}\right)=17-21$, for $N_{\mathrm{HI}}$ in units of $\mathrm{cm}^{-2}$. Contours are drawn at $\log \left(N_{\mathrm{HI}}\right)=17.5,18.5,19,19.5,20, \ldots 21.5$. Note that the HIPASS data is limited by sensitivity to $\log \left(N_{\mathrm{HI}}\right)>18.3$ and the WSRT data to $\log \left(N_{\mathrm{HI}}\right)>17.3$. Kinematic evidence of association with the Magellanic Stream only applies to a subset of the illustrated features (see the text and Fig. 3).

by Putman et al. (2003) based on the HIPASS survey coverage extending to $\delta=+25^{\circ}$. Modeling of the Magellanic Stream (MS) (e.g. Gardiner \& Noguchi 1996; Gardiner 1999) has been moderately successful in reproducing both the distribution and kinematics of the detected gas, but seems to require the inclusion of some ram pressure interaction with the Galactic halo in addition to simply gravitational interaction. Previous models which are dominated by the effects of ram-pressure stripping (Moore \& Davis 1994) do not appear to reproduce the observations as well. The best-fitting tidal-dominated models suggest that the orbit of the Magellanic Clouds with the Galaxy varies between about 150 and $50 \mathrm{kpc}$ radius. The Clouds are currently near peri-galacticon, while the most distant trailing portions of the Stream trace the apo-galacticon portion of the orbit near $125 \mathrm{kpc}$, where the Clouds were located some 0.9 Gyr ago. Ram-pressure dominated models instead suggest that the heliocentric distance is declining along the MS, from $50 \mathrm{kpc}$ at the Clouds to only some $10 \mathrm{kpc}$ at the end of the tail.

We illustrate the combined spatial coverage for the MS in the HIPASS (Putman et al. 2003) and WSRT wide-field surveys in Fig. 5. The LMC and SMC are located in the lower left portion of the image at $(l, b)=(280,-33)$ and $(303,-44)$. The HIPASS coverage and brightness sensitivity are sufficient to detect a continuous filamentary structure beyond a bifurcation near $(l, b)=(80,-65)$ out to a pair of features near $(l, b)=(80,-43)$ and $(95,-50)$. With the order of magnitude greater brightness sensitivity of the WSRT survey, the continuation of each of these filaments can be detected over some additional 10's of degrees. The kinematic continuity of these diffuse features is best assessed in Fig. 3. The westernmost filament in this $(\alpha, \delta)$ depiction is the lower filament of Fig. 5. It extends continuously north to almost $\delta=35^{\circ}$. We will refer to this feature as the "western filament". An additional concentration near $(\alpha, \delta)=(22: 20,+42)$ may or may not form a continuation of this filament. A series of additional features extending in the same general direction as the western filament has radial velocities discrepant by about $200 \mathrm{~km} \mathrm{~s}^{-1}$. The relationship of these additional features to the MS is not clear. The continuation of the upper filament of Fig. 5 is initially due north from $(\alpha, \delta)=(23: 50,+20)$ in Fig. 3. However at $\delta=38^{\circ}$ this filament is observed to loop back to the south-east, leaving the coverage of our WSRT survey at about $(\alpha, \delta)=(01: 00,+20)$. We will refer to this feature as the "eastern loop". Evidence for some of these extensions has been seen previously in the survey of Lockman et al. (2002) in pointed H I observations of 860 semi-random lines-of-sight. Associated OVI absorption from MS extensions in this region has recently been reported by Sembach et al. (2003).

Both the western filament and the eastern loop are conspicuous features of the best-fitting tidal-dominated MS models of Gardiner \& Noguchi (1996) and Gardiner (1999). In these models, such features are near apo-galacticon of the orbit, and correspond to the oldest and most distant $(\sim 125 \mathrm{kpc})$ components of the MS. Since previous observations had not yet detected these features, they must be regarded as predictions of the Gardiner \& Noguchi model; predictions which have been 


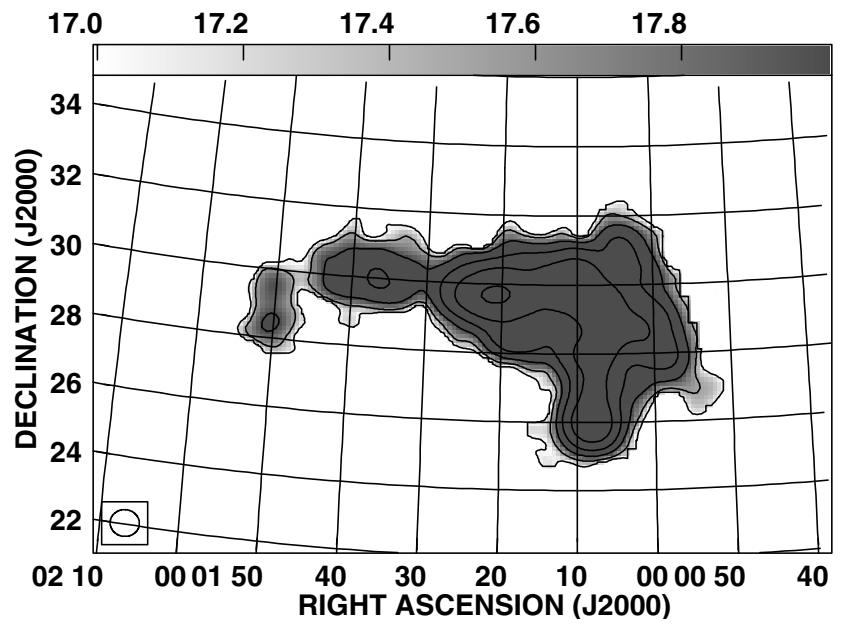

Fig. 6. Integrated H I emission from the subset of detected features apparently associated with Wright's HVC. The grey-scale varies between $\log \left(N_{\mathrm{HI}}\right)=17-18$, for $N_{\mathrm{HI}}$ in units of $\mathrm{cm}^{-2}$. Contours are drawn at $\log \left(N_{\mathrm{HI}}\right)=17,17.5,18, \ldots 19.5$.

amply verified by the current survey results. The models suggest that the radial velocity varies smoothly along the trailing filamentary structures of the MS and that it be essentially single-valued from our perspective. Diffuse, contiguous features appear to conform to these expectations. However, additional discrete features are detected in the vicinity of, and in projection against, the MS at various discrete velocities. There is as yet no evidence to link them to the MS. Existing models do not predict additional features of this type.

\subsection{Wright's cloud}

A striking example of an HVC complex which is located near the Magellanic Stream in projection, but which is kinematically distinct is the feature discovered by Wright (1979). This complex, centered near $(\alpha, \delta)=(01: 15,+29)$, can be seen in Figs. 2 and 3 sandwiched between M 33 to the east and the "eastern loop" feature of the MS, discussed above. The discontinuity in radial velocity between these three different components is apparent in Fig. 3. The features which share kinematic continuity with Wright's Cloud have been isolated in Fig. 6. A faint, newly detected extension of the complex is seen toward the east and south, passing across the southern half of the M 33 disk in projection. The Lockman et al. (2002) survey had also seen indications for extensions of this object.

However, the difference in $V_{\mathrm{Pk}}$ is only a part of what makes Wright's Cloud distinctive. Position-velocity diagrams in (SIN-) projected right ascension and declination (horizontal and vertical in Fig. 2) are shown in Figs. 7 and 8. These perpendicular cross-cuts highlight the remarkable internal kinematics of Wright's Cloud. There appear to be at least two principal kinematic axes in this feature, oriented approximately along PA about $+75^{\circ}$ and $-10^{\circ}$. Displacement along each of these axes is accompanied by a large difference in radial velocity. In the north-south direction the velocity gradient is about $65 \mathrm{~km} \mathrm{~s}^{-1}$ over 3.5 , while in the east-west direction it amounts to about $45 \mathrm{~km} \mathrm{~s}^{-1}$ over 3.7 . Multiple velocity components can be seen along some lines-of-sight. The multi-valued velocity system adjacent to Wright's Cloud (at $(\alpha, \delta)=(00: 50,+30))$ appears to be a continuation of the velocity splitting and eastwest gradient seen in the complex itself. Although this may suggest some physical association of the feature with Wright's Cloud, it was not included in masked version of the data which was integrated to make Fig. 6. Similar velocity gradients along several distinct kinematic axes have been detected previously in the high resolution imaging of CHVC204+30+075 and CHVC115+13-275 by Braun \& Burton (2000). Higher angular resolution imaging of Wright's Cloud may supply insights into the origins of these motions. The presence of such apparently intersecting kinematic systems argues for some form of extreme non-equilibrium phenomenon for their origin, or chance alignment along the line-of-sight.

The relative velocities of M 33 and the Wright's Cloud extension are also apparent in Fig. 7. With our modest spatial resolution these features blend into a semi-continuous distribution, making it difficult to distinguish one from the other.

In the past there has been conjecture regarding the possible association of Wright's Cloud with either M 33 or the Magellanic Stream (Wright 1979). Even after deep, unbiased imaging of the broader environment of Wright's Cloud, we are still not in a good position to assign a likely distance. The complex is moderately distinct from M 33 in position and velocity $\left(\Delta V \sim 200 \mathrm{~km} \mathrm{~s}^{-1}\right)$, while a possible correspondence with the MS might be argued $\left(\Delta V \sim 70 \mathrm{~km} \mathrm{~s}^{-1}\right)$. The integrated H I emission from the Wright Cloud complex is $2800 \mathrm{Jy}-\mathrm{km} \mathrm{s}^{-1}$, corresponding to an $\mathrm{HI}$ mass of $4.6 \times 10^{8} M_{\odot}$ at $840 \mathrm{kpc}$ or $9.5 \times 10^{6} M_{\odot}$ at $120 \mathrm{kpc}$ (if we adopt a distance near apogalacticon in the tidally-dominated MS models). If the previously noted velocity gradients are gravitational in origin we can assess the dark matter fraction for these two assumed distances from the ratio of $R \cdot V^{2} / G \cdot M_{\mathrm{HI}}$. The apparent dark to HI mass ratio (with no inclination correction of measured velocities) varies from 13 at $840 \mathrm{kpc}$ to 95 at $120 \mathrm{kpc}$. The latter possibility in particular merits further consideration, given the similarity in apparent dark fraction found for the discrete HVC population around M 31 by Thilker et al. (2004). We suggest that a plausible explanation of this feature may involve $\mathrm{HI}$ associated with a $10^{9} M_{\odot}$ dark sub-structure within the extended halo of the Galaxy.

Another notable feature which was purposely high-lighted in our choice of cross-cut (in Fig. 7) is the feature we tabulate as CHVC092.6-30.7-404 at $(\alpha, \delta)=(22: 56,+25)$. This is the discrete HVC with the highest velocity width encountered in our sample, with a FWHM line-width of $103 \mathrm{~km} \mathrm{~s}^{-1}$. This feature is seen in projection against the "western filament" of the MS, although it's velocity width sets it apart from any other MS feature detected in the 1800 square degree survey area. If this feature were associated with this portion of the MS, at a likely distance of $120 \mathrm{kpc}$, it would have $M_{\mathrm{HI}}=7.5 \times 10^{5} M_{\odot}$. If the observed line-width of this feature is gravitational then a dark: H I mass ratio of about 1900 is implied. Such a large dark fraction seems quite incredible and suggests that other interpretations for the line-width (or the assumed distance) might be more plausible. 


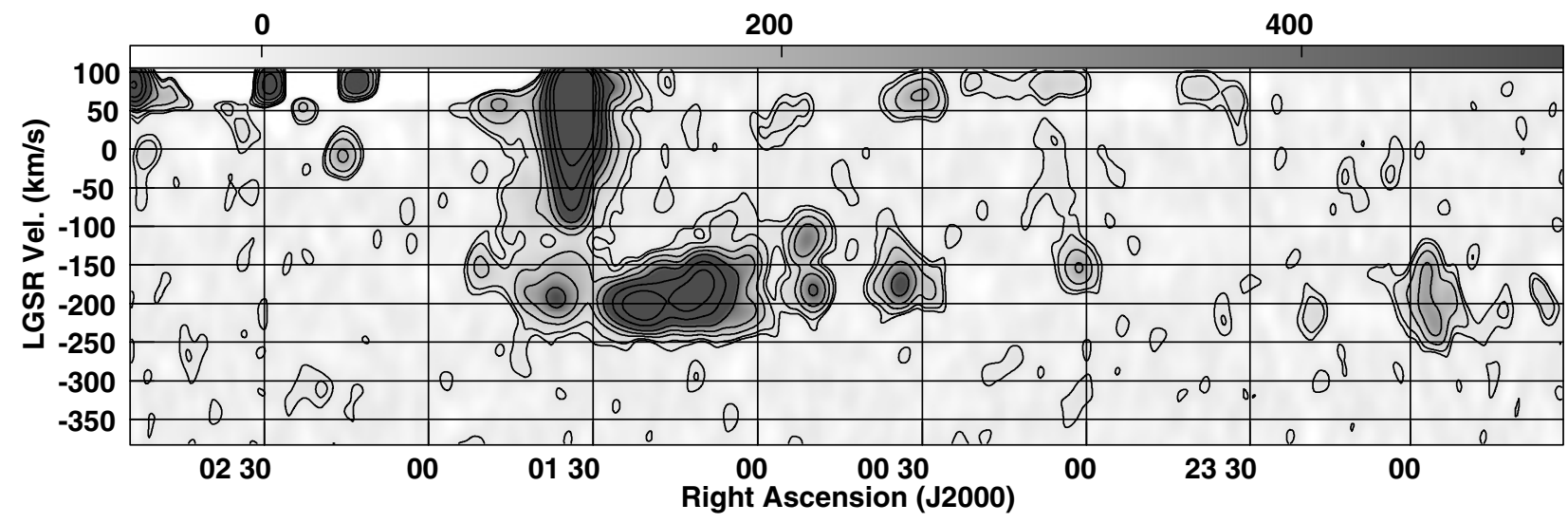

Fig. 7. Position-velocity diagram of the survey region at a central $\delta \sim 30^{\circ}$. The linear grey-scale varies between -50 and $500 \mathrm{mJy} / \mathrm{Beam}$. Contours are drawn at 1, 2, 5, 10, 20, 50, 100 and 200 times $20 \mathrm{mJy} /$ Beam. M 33 is the bright feature at $\alpha=01: 40$, while Wright's Cloud is near $\alpha=01: 15$.



Fig. 8. Position-velocity diagram of the survey region at a central $\alpha=01: 07: 34$. The linear grey-scale varies between -50 and $500 \mathrm{mJy} /$ Beam. Contours are drawn at 1, 2, 5, 10, 20, 50, 100 and 200 times $20 \mathrm{mJy} /$ Beam. Wright's Cloud extends from $\delta=26$ to $30^{\circ}$.

\subsection{The M 31/M 33 filament}

A striking feature of the low column density HI sky is the apparent bridge connecting the systemic velocities of M 31 and M 33. This feature is characterized by peak column densities (at $48^{\prime}$ resolution) of only $\log \left(N_{\mathrm{HI}}\right) \sim 17.5$. We have been able to confirm this exceptionally faint emission with a pointed $30 \mathrm{~min}$. observation with the GBT on 14 June 2003 directed at $(\alpha, \delta)=(01: 20: 29,+37: 33: 33)$. Despite the much smaller GBT beamsize (of only 9 arcmin) the same low column, $\log \left(N_{\mathrm{HI}}\right)=17.5$, was detected at high significance toward this apparent peak in our survey data, suggesting a very diffuse gas distribution.

We have isolated the kinematically continuous features near M 31 and M 33 in Fig. 9. A handful of faint discrete features to the north-east of M 31 are also included in this figure. These features have velocities consistent with an M 31 population (see Fig. 3) but are not kinematically continuous with the diffuse component. Similar discrete features to the south-west of M 31 have been excluded because of the greater chance of confusion with the Magellanic Stream. As noted previously, the bridge can not be traced as far south as M 33, due to foreground confusion from the Galaxy. For the same reason, the

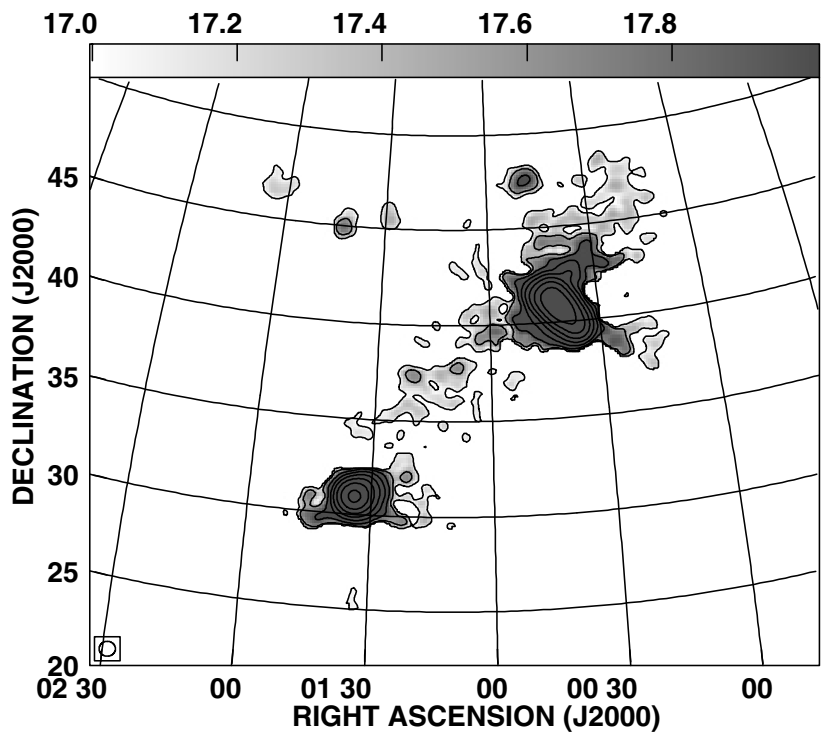

Fig. 9. Integrated H I emission from the subset of detected features apparently associated with M 31 and M 33. The grey-scale varies between $\log \left(N_{\mathrm{HI}}\right)=17-18$, for $N_{\mathrm{HI}}$ in units of $\mathrm{cm}^{-2}$. Contours are drawn at $\log \left(N_{\mathrm{HI}}\right)=17,17.5,18, \ldots 20.5$.

bright disk of M 31 is also partially truncated toward the northeast, giving it a lop-sided appearance relative to the bridge in Fig. 9. Foreground confusion does not hamper detection of the anti-M 33 continuation of the bridge to the north-west of M 31 since the velocity gradient of the bridge is toward more negative LGSR (and GSR) velocity. The total projected extent of this filamentary feature is about $20^{\circ}$, corresponding to $260 \mathrm{kpc}$.

Filamentary components extending between massive galaxies are a conspicuous prediction of high resolution numerical models of structure formation (e.g. Davé et al. 1999, 2001). Such calculations suggest that in the current epoch, cosmic baryons are almost equally distributed by mass amongst three components: (1) galactic concentrations, (2) a warm-hot intergalactic medium (WHIM) and (3) a diffuse intergalactic medium. These three components are each coupled to a decreasing range of baryonic over-density: $\log \left(\rho_{\mathrm{H}} / \bar{\rho}_{\mathrm{H}}\right)>3.5$, 


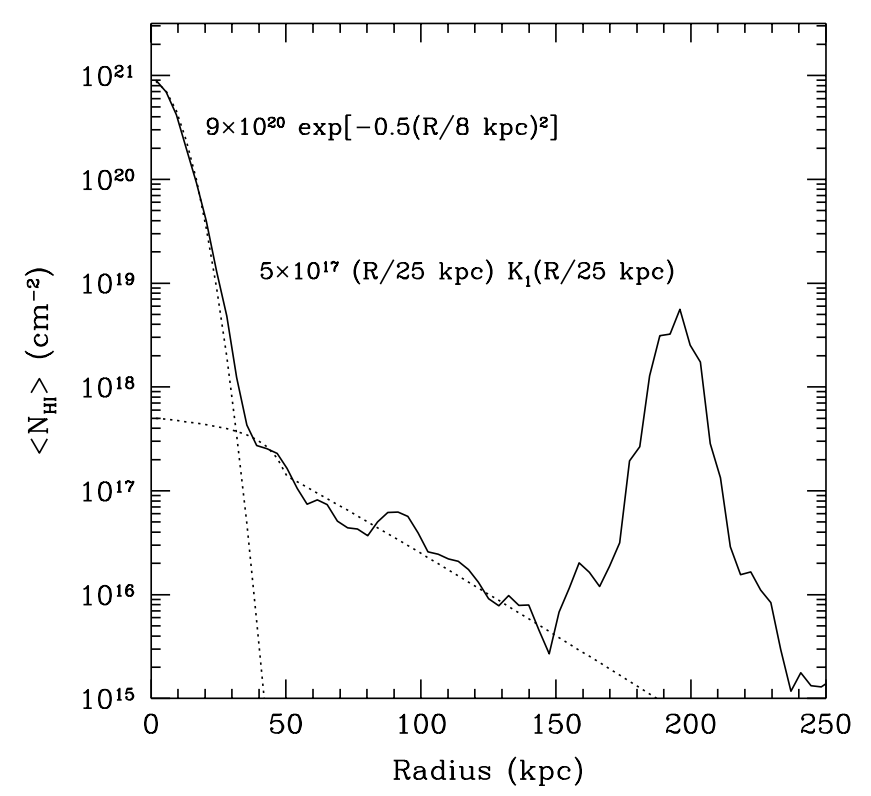

Fig. 10. Azimuthally averaged $\mathrm{H}$ I column density in the vicinity of M 31 as function of radius. The secondary peak near $200 \mathrm{kpc}$ projected distance is due to $\mathrm{M} 33$. A Gaussian of $8 \mathrm{kpc}$ dispersion and peak $\log \left(N_{\mathrm{HI}}\right)=20.95$ provides an adequate description of the central disk. The extended circum-galactic component can be well-fit with a modified Bessel function of $25 \mathrm{kpc}$ scale-length and peak $\log \left(N_{\mathrm{HI}}\right)=17.7$ as discussed in the text.

$1-3.5$, and $<1$ and are probed by QSO absorption lines with specific ranges of neutral column density: $\log \left(N_{\mathrm{HI}}\right)>18,14$ 18 , and $<14$. The neutral fraction is thought to decrease with decreasing column density from about $1 \%$ at $\log \left(N_{\mathrm{HI}}\right)=17$, to less than $0.1 \%$ at $\log \left(N_{\mathrm{HI}}\right)=13$. While a wide range of physical conditions can be found within galaxies, the WHIM is thought to be a condensed shock-heated phase with temperature in the range $10^{5}-10^{7} \mathrm{~K}$, while the diffuse IGM is predominantly photo-ionized with temperature near $10^{4} \mathrm{~K}$. It seems quite conceivable that the M 31/M 33 filament is the neutral manifestation of such a "cosmic web" which contributes to the ongoing fueling of both galaxies. If we assume that the overdensity being traced by this structure is $\log \left(\rho_{\mathrm{H}} / \bar{\rho}_{H}\right) \sim 3$ (cf. Fig. 10 of Davé et al. 1999) then we can calculate the associated baryonic mass from,

$M_{\mathrm{Bar}}=\Omega_{\mathrm{Bar}} \frac{\rho_{\mathrm{H}}}{\bar{\rho}_{\mathrm{H}}} \frac{3 H_{0}^{2}}{8 \pi G} V$

where $V$ is the volume of the structure. Taking $\Omega_{\mathrm{Bar}}=0.03$, $H_{0}=65 \mathrm{~km} \mathrm{~s}^{-1}-\mathrm{Mpc}^{-1}$ and $V=260 \times 26 \times 26 \mathrm{kpc}$ yields $M_{\text {Bar }} \sim 6 \times 10^{8} M_{\odot}$ and presumably $M_{\mathrm{DM}} \sim 6 \times 10^{9} M_{\odot}$. While the apparent baryonic reservoir in such a scenario is quite substantial, the associated dark mass is less than $1 \%$ of that associated with M 31 .

A second scenario for the origin of this feature might be a major tidal interaction between M 31 and M 33 at some time in the past. If we adopt distances, $D_{\mathrm{M} 31}=790$ and $D_{\mathrm{M} 33}=840 \mathrm{kpc}$ (Freedman et al. 2001), then the physical separation of the two systems is $218 \mathrm{kpc}$ and the angle between a radial vector connecting M 33 to the Sun with the vector connecting M 33 and M 31 is about $70^{\circ}$. If we assume, as seems plausible from test particle simulations in the Local Group potential (eg. Blitz et al. 1999), that the true space velocity of M 33 is directed in the (anti-)M 31 direction, then we can estimate it's sign and magnitude from the observed radial velocity along the M 33 to Sun vector. The measured systemic velocities of M 31 and M 33 in various reference frames are summarized in Table 1. The GSR velocity of M 33, $V_{\mathrm{GSR}}=-44 \mathrm{~km} \mathrm{~s}^{-1}$, is the relevant one, given our heliocentric perspective. It seems likely that M 33 is approaching M 31 with a velocity of about $44 / \cos \left(70^{\circ}\right)=130 \mathrm{~km} \mathrm{~s}^{-1}$. This would seem to argue against even a moderately recent (within $2 \mathrm{Gyr}$ ) interaction for which a receding velocity might have been an indication.

\subsection{The M 31 HVC populations}

A major step forward in delineating the M $31 \mathrm{HVC}$ populations was made in our GBT survey (Thilker et al. 2004). Evidence was found for three distinct components of high velocity $\mathrm{HI}$ within the $95 \times 95 \mathrm{kpc}$ field imaged with the GBT: (1) several features were identified of likely tidal origin, including a filamentary component having partial spatial correspondence with the "giant" stellar stream (Ibata et al. 2001; Ferguson et al. 2002, McConachie et al. 2003). (2) A "halo" component of diffuse filamentary features was detected concentrated at the M 31 systemic velocity. (3) A population of about 20 discrete $\mathrm{HVC}$ features was detected within the $7 \times 7^{\circ}$ survey region. The discrete HVC's roughly follow the kinematic pattern of outer disk rotation and display a correlation of apparent H I mass with internal line-width, consistent with a dark- to neutral gas-mass ratio of about 100:1.

All three of these HVC components are apparent in our wide-field survey, albeit with reduced point-source sensitivity and angular resolution. The much wider field-of-view and higher brightness sensitivity for diffuse features of the current work has permitted several important additional conclusions to be drawn regarding the HVC population of M 31: (1) The extreme velocity range of discrete features (comparable with M 31 rotation) is only detected within about $12^{\circ}$ (corresponding to $160 \mathrm{kpc}$ ) of M 31, despite a much larger FOV over which such features could have been seen. (2) The systemic velocity "halo" component appears to extend into a diffuse bridge connecting the systemic velocities of M 31 and M 33, while continuing in the anti-M 33 direction for an additional $\sim 150 \mathrm{kpc}$. (3) The M 31 HVC populations appear to be morphologically and kinematically distinct from the Magellanic Stream, although there is undoubtedly some confusion of discrete features which happen to lie toward the "eastern loop".

It is instructive to consider what the expected properties might be of a circum-galactic population of low-mass dark-matter halos. Sternberg et al. (2002) have calculated the hydrostatic and ionization structures for such objects under a variety of assumptions about the confining medium. Bound neutral cores can only be realized with dark matter masses larger than about $M_{\mathrm{DM}}>10^{8} M_{\odot}$, corresponding to maximum rotation velocities, $v_{\max }>10 \mathrm{~km} \mathrm{~s}^{-1}$. This is because a shielding column of warm ionized gas (WIM, with temperature $10^{4} \mathrm{~K}$ ) must be retained, before a warm neutral component 
(WNM, with $8000<T<10^{4} \mathrm{~K}$ ) can survive. Under appropriate conditions, a cool neutral core (CNM, with $50<T<$ $200 \mathrm{~K}$ ) can condense within this nested structure. The H I mass associated with these low mass objects depends sensitively on the external pressure, $P_{\mathrm{HIM}} / k$, of the hot ionized gas (HIM, with $10^{5.5}<T<1^{6.5} \mathrm{~K}$ ) within which they are embedded. For $M_{\mathrm{DM}}=10^{8} M_{\odot}, M_{\mathrm{HI}}$ varies approximately quadratically from about $10^{3}$ to $10^{7} M_{\odot}$ with the external pressure, $P_{\mathrm{HIM}} / k=1$ to $100 \mathrm{~cm}^{-3} \mathrm{~K}$. For a realistic external pressure of a few 10's of $\mathrm{cm}^{-3} \mathrm{~K}$, the minimum expected H I mass might be about $10^{5} M_{\odot}$. Given the steeply rising mass function at low $M_{\mathrm{DM}}$, $N\left(M_{\mathrm{DM}}\right) \propto M_{\mathrm{DM}}^{-2}$, this will also be the most commonly occurring H I mass. In their Eq. (38), Sternberg et al. give the expected number of sub-halos within a distance, $d$, of the parent halo of mass, $M_{\mathrm{vir}, p}$, which exceed a given $v_{\max }$, based on the numerical simulations of Klypin et al. (1999),

$$
\begin{aligned}
N\left(>v_{\max },<d\right)=1.06 \times 10^{3} & \left(\frac{M_{\mathrm{vir}, p}}{10^{12} M_{\odot}}\right) \\
& \times\left(\frac{v_{\max }}{8 \mathrm{~km} \mathrm{~s}^{-1}}\right)^{-2.75}\left(\frac{d}{1 \mathrm{Mpc}}\right) .
\end{aligned}
$$

Thilker et al. (2004) have already pointed out that the 20 discrete objects with H I masses in the range $10^{5}$ and $10^{7} M_{\odot}$ detected in the GBT survey field are in moderately good agreement with the expected number, $N=25$, predicted by the above equation (with $v_{\max }>10 \mathrm{~km} \mathrm{~s}^{-1}, M_{\mathrm{vir}, p}=10^{12} M_{\odot}$ and $d=40 \mathrm{kpc}$ ). The $8 \sigma$ completeness limit of the GBT survey (for a $15^{\prime}, 32 \mathrm{~km} \mathrm{~s}^{-1}$ source extent) is $2 \times 10^{5} M_{\odot}$ at the M 31 distance, suggesting that the GBT detections are already quite incomplete at the low mass end. The $8 \sigma$ completeness limit of the WSRT wide-field survey (for a spatially unresolved, $32 \mathrm{~km} \mathrm{~s}^{-1}$ source extent) is $4.5 \times 10^{5} M_{\odot}$. The relative completeness of the current and the GBT surveys is confirmed within the $7 \times 7^{\circ}$ region of overlapping coverage. Only the brighter (and unconfused) GBT detections are apparent in our wide-field survey.

Extending the survey coverage from $d=40 \mathrm{kpc}$, probed by our GBT data, to $d=160 \mathrm{kpc}$ in our wide-field data is predicted (by Eq. (5)) to increase the number of such objects by a factor of four to about 100 . However, the implication of our moderately high completeness limit on large scales, $M_{\mathrm{HI}}=$ $4.5 \times 10^{5} M_{\odot}$, is that such a population of low mass discrete components will require higher sensitivity for their detection. This would be exacerbated in practise by a likely radial gradient in $P_{\mathrm{HIM}}$ in the extended halo of M 31. A decline in $P_{\mathrm{HIM}}$ at larger radii would result in a rapid decline in the associated $\mathrm{HI}$ mass of any dark matter sub-halo.

Although our sampling of the HVC population of M 31 is known to be incomplete, due to the effects of confusion with both the Galaxy and the Magellanic Stream, as well as our limited sensitivity, we can begin to assess the properties of the combined large-scale distribution. In Fig. 10 we plot the azimuthally averaged column density of currently detected features as a function of projected distance to M 31. Only the subset of features apparently associated with M 31 and M 33 (and illustrated in Fig. 9) have been used in making this figure. The bright central disk component can be characterized by a Gaussian of $8 \mathrm{kpc}$ dispersion and peak column density $\log \left(N_{\mathrm{HI}}\right)=20.95$. The secondary peak in the figure near $200 \mathrm{kpc}$ radius is due to the bright disk emission of M 33. The distribution of circum-galactic gas can be well-described by the projection of a radial exponential of the neutral gas volume density, $n_{\mathrm{HI}}$, with a radial scale-length, $h=25 \mathrm{kpc}$ and a peak $\log \left(N_{\mathrm{HI}}\right)=17.7$. Taking

$n_{\mathrm{HI}}(r)=n_{\mathrm{o}} \mathrm{e}^{-r / h}$

in terms of the radial distance, $r$; the corresponding projected distribution of H I column density,

$N_{\mathrm{HI}}(r)=2 h n_{\mathrm{o}} \frac{r}{h} K_{1}\left(\frac{r}{h}\right)$

where $K_{1}$ is the modified Bessel function of order 1 . The peak column density of the distribution is $N_{\mathrm{HI}}(0)=2 h n_{\mathrm{o}}$ and the integrated mass is given by,

$M_{\mathrm{HI}}=24 \pi n_{\mathrm{o}} m_{\mathrm{HI}} h^{3}=12 \pi m_{\mathrm{HI}} N_{\mathrm{HI}}(0) h^{2}$.

The projected-exponential (of Eq. (7)) provides a better fit to the observed profile than a simple exponential or Gaussian, particularly for the "shoulder" feature near $50 \mathrm{kpc}$, where much of the mass of the distribution is concentrated. This form has the additional advantage (over a 1-D exponential, for example) of lending itself to a straightforward physical interpretation. The integrated H I mass of the disk Gaussian component is $2.9 \times 10^{9} M_{\odot}$ and of the circum-galactic exponential is $9.4 \times 10^{7} M_{\odot}$. The disk component falls about $40 \%$ short of the total H I mass of M 31 as discussed further below.

Although the circum-galactic component of M 31 is spatially quite extended, it is more centrally concentrated than the best-fitting models for a Galactic sub-halo population of De Heij et al. (2002c), which called for a Gaussian spatial dispersion between about 150-200 kpc. Those model fits were constrained by the observed properties of the allsky CHVC samples of Putman et al. (2002) and De Heij et al. (2002a) which appear to be completely dominated by the sub-population associated with the Galaxy. In predicting the attributes of a corresponding M 31 population, De Heij et al. assumed that identical spatial scale-lengths might apply to both the Galaxy and M 31, and that the total number of M 31 subhalos would be about twice that associated with the Galaxy, given a factor two higher assumed virial mass (cf. Eq. (5)). It is conceivable that the circum-galactic populations of the Galaxy and M 31 are actually quite different and also that the distributions are not spherically symmetric, but rather more filamentary in character as is quite apparent from Fig. 9.

In Fig. 11 we plot the cumulated H I mass due to linesof-sight with less than the indicated column density. The distribution depicted in Fig. 9 leads to the solid line in the figure. For comparison we also plot the cumulated mass detected in our complimentary high resolution survey (Braun et al. 2002) of the central $80 \times 40 \mathrm{kpc}$ (major $\times$ minor axis) area as a dashed line. The apparent rapid roll-off in the curves below about $\log \left(N_{\mathrm{HI}}\right)=17.2$ (for the wide-field survey) and $\log \left(N_{\mathrm{HI}}\right)=19.2$ (for the high-resolution survey) is an artifact of the limited sensitivity. The solid curve only reaches about $60 \%$ of the total mass given by the dashed curve, since the integration in velocity has been truncated where foreground confusion 


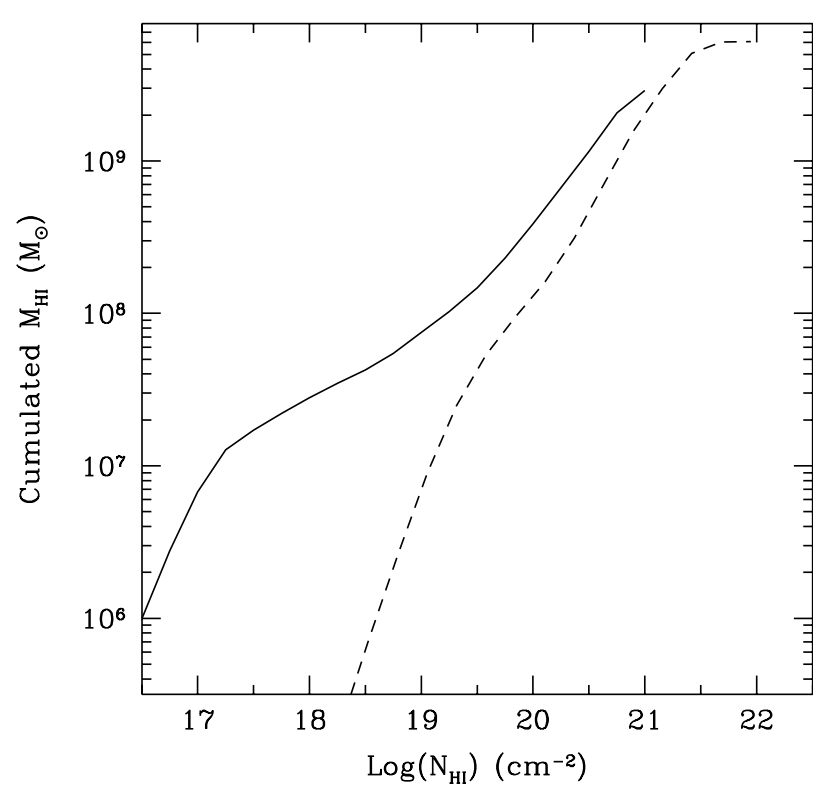

Fig. 11. Cumulated H I mass in the vicinty of M 31 below the indicated column density. The solid line is taken from the current wide-field survey for the features depicted in Fig. 9. The dashed line is taken from our high resolution mosaic (Braun et al. 2002) of the inner 80 by $40 \mathrm{kpc}$.

sets in for the wide-field data. This truncation could be circumvented in the high-resolution data by careful spatial filtering. The excess H I mass due to low column density emission in the extended M 31 environment (out to $160 \mathrm{kpc}$ ) amounts to about $10^{8} M_{\odot}$. This is in good agreement with the integral of the projected exponential function noted above. Relative to a total $M_{\mathrm{HI}}$ of about $5 \times 10^{9} M_{\odot}$, this is only about $2 \%$.

\subsection{Circum-galactic HI and QSO absorption lines}

In addition to the current wide-field survey of the M 31 environment $(800 \times 400 \mathrm{kpc}$ at $11 \mathrm{kpc}$ resolution), we have imaged the central $95 \times 95 \mathrm{kpc}$ with the GBT (Thilker et al. 2004) at $2 \mathrm{kpc}$ resolution and the central $80 \times 40 \mathrm{kpc}$ (major $\times$ minor axis) area at up to $50 \mathrm{pc}$ resolution in our WSRT synthesis mosaic (Braun et al. 2002, 2003). The combined database of HI detections associated with M 31 enables compilation of the distribution function of H I column density spanning an unprecedented range of almost five orders of magnitude from $\log \left(N_{\mathrm{HI}}\right)=17.2$ to $\log \left(N_{\mathrm{HI}}\right)=21.9$. We plot the combined distribution function in Fig. 12, where the dashed line is drawn from our synthesis mosaic at $1^{\prime}$ resolution, the dotted and solid lines from our GBT survey and the dot-dash line from the current work. The distinction between the dotted and solid curves for the GBT data is that all features are included in the solid line plot, while only the peculiar velocity features that could be clearly isolated from normal disk emission are indicated by the dotted line. Each curve displays an apparent turn-over at low column densities which reflects the finite column density sensitivity of the survey. For our synthesis mosaic data this sets in below $\log \left(N_{\mathrm{HI}}\right)=19.3$, for the GBT survey below about $\log \left(N_{\mathrm{HI}}\right)=18$ and for the wide-field survey below about

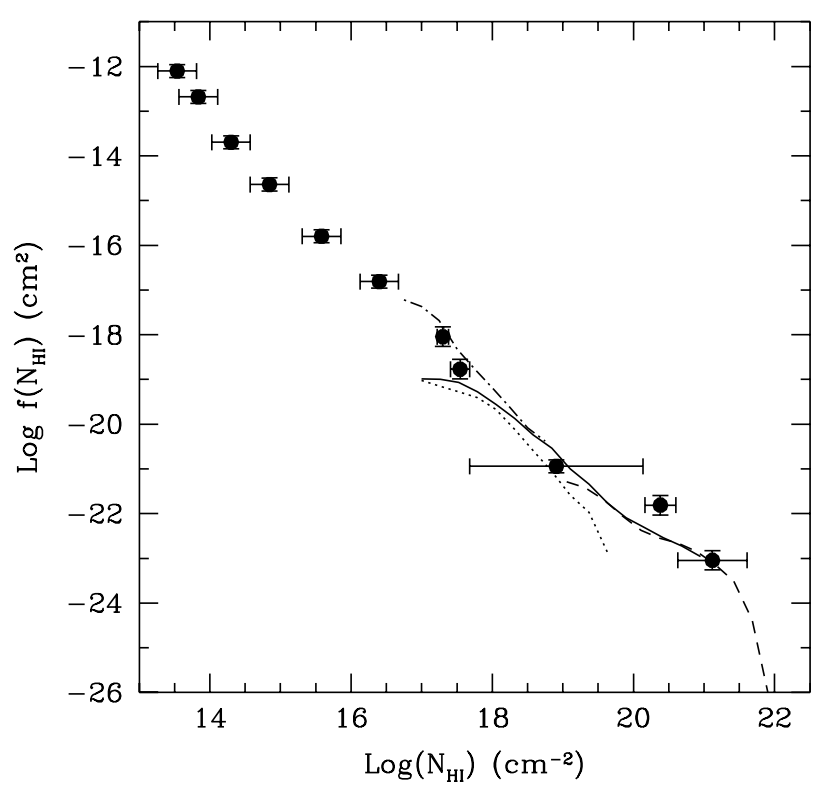

Fig. 12. The distribution function of $\mathrm{H}$ I column density due to M 31 and it's environment. The data from three Hi surveys of M 31 are combined in this figure to probe column densities over a total range of some five orders of magnitude. The dashed line is from the WSRT mosaic (Braun et al. 2003) with 1' resolution over $80 \times 40 \mathrm{kpc}$, the dotted and solid lines from our GBT survey (Thilker et al. 2004) with $9^{\prime}$ resolution over $95 \times 95 \mathrm{kpc}$ and the dot-dash line from the current work with $48^{\prime}$ resolution out to $150 \mathrm{kpc}$ radius. The filled circles with errorbars are the low red-shift QSO absorption line data as tabulated by Corbellli \& Bandiera (2002).

$\log \left(N_{\mathrm{HI}}\right)=17$. The milder flattening of the GBT data below $\log \left(N_{\mathrm{HI}}\right)=18.2$ is very likely due to an insufficient field-ofview to fully sample the distribution.

The curves in Fig. 12 were calculated from,

$f\left(N_{\mathrm{HI}}\right)=\frac{c}{H_{0}} \frac{\rho_{\mathrm{av}}}{\rho_{\mathrm{M} 31}} \theta_{\mathrm{M} 31} \frac{A\left(N_{\mathrm{HI}}\right)}{d N_{\mathrm{HI}}} \mathrm{cm}^{2}$

where

$\theta_{\mathrm{M} 31}=\theta_{*} \ln (10)\left(M_{\mathrm{M} 31} / M_{*}\right)^{\alpha+1} \exp \left(-M_{\mathrm{M} 31} / M_{*}\right)$

and $\rho_{\mathrm{M} 31}=\theta_{\mathrm{M} 31} M_{\mathrm{M} 31} \cdot A\left(N_{\mathrm{HI}}\right)$ is the surface area subtended by $\mathrm{HI}$ in the column density interval $d N_{\mathrm{HI}}$ centered on $N_{\mathrm{HI}}$. The HIMF parameters were taken from Zwaan et al. (2003); namely a faint-end slope, $\alpha=-1.30$, characteristic H I mass, $\log \left(M_{*} / M_{\odot}\right)=9.79$ and normalization, $\theta_{*}=8.6 \times 10^{-3} \mathrm{Mpc}^{-3}$, where $H_{0}=75 \mathrm{~km}^{-1} \mathrm{Mpc}^{-1}$ has been assumed throughout. The term, $\rho_{\mathrm{av}} / \rho_{\mathrm{M}} 31$ in Eq. (9) is introduced to normalize our measurements for a single galaxy, M 31, to the global average density of $\mathrm{HI}$ in galaxies, $\rho_{\mathrm{av}}=6.1 \times 10^{7} h_{75} M_{\odot} \mathrm{Mpc}^{-3}$, also taken from Zwaan et al. Since the H I mass of M 31 places it close to $M_{*}$, this is a relatively small upward correction of only $30 \%$.

Our distribution function can be compared with previous H I work by Ryan-Weber et al. (2003) and Rao \& Briggs (1993) who considered H I selected samples of galaxies. While these studies are superior in their sampling of a range in Galaxy type and HI mass, they are limited by sensitivity to about 
$\log \left(N_{\mathrm{HI}}\right)>19.5$ and by angular resolution to $\log \left(N_{\mathrm{HI}}\right)<21$. Of course, for $\log \left(N_{\mathrm{HI}}\right)>21$ there is a high liklihood of significant optical depth in the H I emission line (Braun \& Walterbos 1992; Braun 1997), so that even our high resolution estimates must be regarded as lower limits in this column density range.

We also compare our distribution function to the low redshift QSO absorption line data, as tabulated by Corbelli \& Bandiera (2002), in Fig. 12. The filled circles with error bars show the QSO data together with the column density ranges over which they were defined. Four of the five measurement points show very good agreement with our distribution function. Only the QSO measurement near $\log \left(N_{\mathrm{HI}}\right)=20.4$ suggests an excess probability of absorption by more than a factor of four relative to that seen toward M 31. The H I distribution function of Ryan-Weber et al. (2003) is in good agreement with ours in this column density range, making it unlikely that this apparent deficit is simply a peculiarity of the H I distribution of M 31. An explanation for this apparent discrepancy may lie in the misclassification of some absorption line systems (e.g. Turnshek \& Rao 2002). In Fig. 12 we plot the low red-shift optical distribution function over it's entire measured range, to put our result into a broader perspective. If the distribution we detect around M 31 is at all representative, then it can account very well for the QSO absorption line statistics.

The QSO absorption line data has a profound implication for studies of the H I universe. By going down in column density sensitivity from $\log \left(N_{\mathrm{HI}}\right)=19$, such as available in the deepest current imaging studies of nearby galaxies, to $\log \left(N_{\mathrm{HI}}\right)=17$, the surface covering factor of $\mathrm{HI}$ emission features should increase by about a factor of 30 . The QSO absorption line statistics allow this prediction to be made with great confidence. There has been debate about how this gas might be distributed, but it seemed intimately linked in some way to $\mathrm{L}_{*}$ (and therefore $M_{*}$ ) galaxies, possibly in the form of a cloud of low-mass satellites or an extension of the $\mathrm{L}_{*}$ galaxy disk. In at least one instance we have been able to confirm this predicted increase in surface covering factor at low $N_{\mathrm{HI}}$ and demonstrate in an image how the system is related to the host Galaxy. As noted above, the detected morphology is suggestive of the "cosmic web" of filaments which are predicted in recent numerical simulations of structure formation (e.g. Davé et al. 1999).

\section{Summary}

Our wide-field H I survey of $1800 \mathrm{deg}^{2}$ around M 31 has provided a number of insights into the low $N_{\mathrm{HI}}$ sky. Peculiar velocity neutral gas, in excess of our $3 \sigma$ limit of $N_{\mathrm{HI}}=1.5 \times$ $10^{17} \mathrm{~cm}^{-2}$, is seen from $29 \%$ of our survey area. This is comparable to the $37 \%$ detection rate of Lockman et al. (2002) for high velocity gas, with $N_{\mathrm{HI}}>3 \times 10^{17} \mathrm{~cm}^{-2}$, toward 860 semirandom directions at $\delta>-44^{\circ}$.

Our improved sensitivity over previous surveys has permitted detection of an order of magnitude more discrete HVC features in this region, increasing their number from 9 to 95 . The vast majority of these discrete features $(83 \%)$ are isolated in position and velocity at a level of $N_{\mathrm{HI}}=1.5 \times 10^{17} \mathrm{~cm}^{-2}$ from any adjoining HVC complexes, and may thus be termed CHVCs. While the distributions of angular size and velocity width of these features are comparable to what has been seen previously, the distribution of flux density appears to have an inflection point from a steep power-law index (-2.15 in linear units) above $12 \mathrm{Jy}-\mathrm{km} \mathrm{s}^{-1}$ to a much flatter distribution down to our $8 \sigma$ completeness limit of $6 \mathrm{Jy}-\mathrm{km} \mathrm{s}^{-1}$ (see Fig. 4). Such an inflection point may be an indication that the spatial distribution of discrete HVCs is not isotropic. Sternberg et al. (2002) have already pointed out that the total number of cataloged CHVCs (in the HIPASS and LDS samples) is consistent with that expected from the numerical $\Lambda \mathrm{CDM}$ simulations and parameterized in Eq. (5) if the population has a characteristic distance of about $150 \mathrm{kpc}$. At such a characteristic distance, $12 \mathrm{Jy}-\mathrm{km} \mathrm{s}^{-1}$ corresponds to about $M_{\mathrm{HI}}=6 \times 10^{4} M_{\odot}$.

A prominent diffuse feature detected in our survey field is a northern extension of the Magellanic Stream, as seen in Fig. 5. At northern declinations the MS is bifurcated into a linear feature which we term the "western filament" as well as an arc-like structure we term the "eastern loop". Typical column densities in these features are about $3 \times 10^{17} \mathrm{~cm}^{-2}$, while the line-of-sight velocity is essentially single-valued and varies smoothly with position as shown in Fig. 3. Both the location and kinematics of these extensions agree well with the tidally-dominated simulations of Gardiner \& Noguchi (1996) and Gardiner (1999). In these models the "eastern loop" corresponds to the apogalacticon portion of the LMC/SMC orbit, near $125 \mathrm{kpc}$, where the clouds were located some 0.9 Gyr previously.

Adjacent to the MS is the HVC feature known as Wright's Cloud. We detect faint extensions of this complex reaching about $6^{\circ}$ to the south-east and high-light the peculiar internal kinematics of the bright core region. Two principal kinematic axes are present which are essentially perpendicular. Velocity gradients of at least 65 and $45 \mathrm{~km} \mathrm{~s}^{-1}$ are present along these overlapping axes. We suggest that a plausible explanation of this feature may involve an $\mathrm{H}$ I condensation of about $10^{7} M_{\odot}$ on a dark sub-structure of $10^{9} M_{\odot}$ in the extended galactic halo at a distance of about $120 \mathrm{kpc}$. Higher resolution imaging should help to better constrain the kinematics and likely origin of this source.

In the immediate vicinity of M 31 we detect a system of very faint discrete $\mathrm{H}$ I features with radial velocities that span most of the range corresponding to rotation in the M 31 disk. Such features are only detected out to a radius of about $12^{\circ}$, corresponding to $160 \mathrm{kpc}$ from M 31 with our current sensitivity (an $8 \sigma$ completeness limit of $4.5 \times 10^{5} M_{\odot}$ ). The inner $95 \times 95 \mathrm{kpc}$ of this distribution has been imaged with higher resolution and point source sensitivity in our complimentary survey with the GBT (Thilker et al. 2004) (for which the $8 \sigma$ completeness limit is $\left.2 \times 10^{5} M_{\odot}\right)$. With the higher resolution of the GBT ( $9^{\prime}$ corresponding to $2 \mathrm{kpc}$ ) it is clear that a subset of these discrete features are tidal in origin, while others do not appear to be. Only one feature in this class (Davies' Cloud) has an apparent $\mathrm{HI}$ mass as high as $10^{7} M_{\odot}$, with the remainder in the range $10^{5} \rightarrow 10^{6} M_{\odot}$. This discrete population appears to be an excellent candidate for the $\mathrm{H}$ I associated with low mass dark matter satellites of M 31. The detected numbers of objects and their associated H I mass are in good agreement with expectations (Sternberg et al. 2002). 
A diffuse filamentary distribution of $\mathrm{HI}$ is detected at the systemic velocity of M 31 . The filament extends in the direction of M 33 (at a projected distance of $200 \mathrm{kpc}$ ), varying in line-ofsight velocity toward the M 33 systemic velocity. The filament is also detected out to about $150 \mathrm{kpc}$ in the anti-M 33 direction. Peak column densities in this feature amount to only about $3 \times$ $10^{17} \mathrm{~cm}^{-2}$ in an $11 \mathrm{kpc}$ beam. A similarly low column density has been detected in a deep pointed observation of a local peak with the much smaller GBT beam $(2 \mathrm{kpc})$, suggesting that the filament is indeed extremely diffuse. This feature appears to be an extension of the "halo" component concentrated at the M 31 systemic velocity that was seen in our GBT survey (Thilker et al. 2004). This structure is suggestive of H I associated with a filament of coronal gas, either in the form of localized neutral condensations are simply as a trace neutral constituent of gas with a high degree of ionization. If we are detecting $\mathrm{H}$ I from a warm-hot inter-galactic medium (WHIM) filament then it is likely to contain some $6 \times 10^{8} M_{\odot}$ of baryons and presumably $6 \times 10^{9} M_{\odot}$ of dark matter.

The distribution of $\mathrm{HI}$ in the extended environment of M 31 can be described by a projected exponential function $\left(x K_{1}(x)\right)$ of $25 \mathrm{kpc}$ scale-length and $5 \times 10^{17} \mathrm{~cm}^{-2}$ peak column density. The combined distribution function of $\mathrm{H} \mathrm{I}$ in the M 31 disk and environment has been constructed over the column density range $\log \left(N_{\mathrm{HI}}\right)=17.2$ to $\log \left(N_{\mathrm{HI}}\right)=21.9$ by utilizing all three of our complimentary surveys spanning resolutions of $50 \mathrm{pc}$ to $11 \mathrm{kpc}$. The composite distribution function provides very good agreement with the low red-shift QSO absorption line data (as tabulated by Corbelli \& Bandieri 2002) over this entire range, with the exception of the QSO point near $\log \left(N_{\mathrm{HI}}\right)=20.4$, for which the M 31 data fall short by about a factor of four.

Our survey has demonstrated that the predicted increase in surface covering factor of $\mathrm{HI}$ at low column density (by more than three orders of magnitude) implied by the QSO absorption line statistics is borne out in practise. Extremely diffuse filaments of the "cosmic web" in the environment of $M_{*}$ galaxies are open to direct imaging with sensitive observations of the $\lambda 21 \mathrm{~cm}$ line.

Acknowledgements. We thank Jay Lockman for obtaining the confirming GBT spectrum of the M 31/M 33 filament, Frank Briggs for useful discussions concerning normalization methods of $\mathrm{HI}$ distribution functions and Mary Putman for making a digital version of the HIPASS image of the Magellanic Clouds and Stream available to us. The Westerbork Synthesis Radio Telescope is operated by the Netherlands Foundation for Research in Astronomy under contract with the Netherlands Organization for Scientific Research.

\section{References}

Barnes, D. G., Staveley-Smith, L., de Blok, W. J. G., et al. 2001, MNRAS, 322, 486

Blitz, L., Spergel, D. N., Teuben, P. J., et al. 1999, ApJ, 514, 818

Braun, R., \& Walterbos, R. A. M. 1992, ApJ, 386, 120

Braun, R. 1997, ApJ, 484, 637
Braun, R., \& Burton, W. B. 1999, A\&A, 341, 437

Braun, R., \& Burton, W. B. 2000, A\&A, 354, 853

Braun, R., Thilker, D. A., Corbelli, E., \& Walterbos, R. A. M. 2002, http://www. astron.nl/newsletter/2002-1/index.html

Braun, R., Thilker, D. A., \& Walterbos, R. A. M. 2003, A\&A, 406, 829

Braun, R., Thilker, D. A., Corbelli, E., \& Walterbos, R. A. M. 2003, $A \& A$, in preparation

Burton, W. B., Braun, R., \& Chengalur, J. N. 2001, A\&A, 369, 616

Corbelli, E., \& Bandiera, R. 2002, ApJ, 567, 712

Davé, R., Hernquist, L., Katz, N., \& Weinberg, D. H. 1999, ApJ, 511, 521

Davé, R., Cen, R., Ostriker, J. P., et al. 2001, ApJ, 552, 473

Davies, R. D. 1975, MNRAS, 170, 45P

de Heij, V., Braun, R., \& Burton, W. B. 2002a, A\&A, 391, 159

de Heij, V., Braun, R., \& Burton, W. B. 2002b, A\&A, 391, 67

de Heij, V., Braun, R., \& Burton, W. B. 2002c, A\&A, 392, 417

Ferguson, A. M. N., Irwin, M. J., Ibata, R. A., et al. 2002, AJ, 124, 1452

Freedman, W. M., Madore, B. F., \& Gibson, B. K., et al. 2001, ApJ, 553, 47

Gardiner, L. T., \& Noguchi, M. 1996, MNRAS, 278, 191

Gardiner, L. T. 1999, in High-Velocity Clouds, ed. B. Gibson, \& M. Putman (San Francisco: ASP), ASP Conf. Ser. 166, 292

Gooch, R. 1995, in Astronomical Data Analysis Software and Systems IV, ed. R. A. Shaw, H. E. Payne, \& J. E. Haynes (San Francisco: ASP), ASP Conf. Ser., 77, 144

Hartmann, D., \& Burton, W. B. 1997, Atlas of Galactic Neutral Hydrogen (Cambridge University Press)

Hulsbosch, A. N. M. 1978, A\&A, 66, L5

Ibata, R., Irwin, M., Lewis, G., et al. 2001, Nature, 412, 49

Klypin, A., Kravtsov, A. V., Valenzuela, O., \& Prada, F. 1999, ApJ, 522,82

Lockman, F. J., Murphy, E. M., Petty-Powell, S., \& Urick, V. J. 2002 , ApJS, 140, 331

Mathewson, D. S., Cleary, M. N., \& Murray, J. D. 1974, ApJ, 190, 291

McConnachie, A. W., Irwin, M. J., Ibata, R. A., et al. 2003, MNRAS, 343,1335

Moore, B., Davis, M. 1994, MNRAS, 270, 209

Moore, B., Ghigna, S., Governato, F., et al. 1999, ApJ, 524, L19

Muller, C. A., Oort, J. H., \& Raimond, E. 1963, C. R. Acad. Sci. Paris, 257,1661

Putman, M. E., de Heij, V., Stavely-Smith, L., et al. 2002, AJ, 123, 873

Putman, M. E., Stavely-Smith, L., Freeman, K., et al. 2003, ApJ, 586, 170

Rao, S., \& Briggs, F. 1993, ApJ, 419, 515

Rosenberg, J. L., \& Schneider, S. E. 2002, ApJ, 567, 247

Ryan-Weber, E. V., Webster, R. L., \& Staveley-Smith L. 2003, MNRAS, 343, 1195

Sembach, K. R., Wakker, B. P., Savage, B. D., et al. 2003, ApJS, 146, 165

Sternberg, A., McKee, C. F., \& Wolfire, M. G. 2002, ApJS, 143, 419

Thilker, D. A., Braun, R., Walterbos, R. A. M., et al. 2004, ApJ, 601, L39

Turnshek, D. A., \& Rao, S. M. 2002, ApJ, 572, L7

Wakker, B. P., \& van Woerden, H. 1991, A\&A, 250, 509

Wright, M. C. H. 1979, ApJ, 233, 35

Zwaan, M. A., Staveley-Smith L., Koribalski, B. S., et al. 2003, AJ, 125,2842 